\title{
Defining severe obstructive lung disease in the biologic era: an endotype-based approach
}

\author{
Richard J. Martin ${ }^{1}$, Elisabeth H. Bel ${ }^{2}$, Ian D. Pavord ${ }^{3}$, David Price $\mathbb{1}^{4,5}$ and \\ Helen K. Reddel $\mathbb{1}^{6}$
}

Affiliations: ${ }^{1}$ National Jewish Health and the University of Colorado, Denver, CO, USA. ${ }^{2}$ Amsterdam University Medical Centre, University of Amsterdam, Amsterdam, the Netherlands. ${ }^{3}$ Respiratory Medicine Unit and NIHR Oxford Respiratory BRC, Nuffield Dept of Medicine, University of Oxford, Oxford, UK. ${ }^{4}$ Observational and Pragmatic Research Institute, Singapore. ${ }^{5}$ Centre of Academic Primary Care, University of Aberdeen, Aberdeen, UK. ${ }^{6}$ Woolcock Institute of Medical Research, University of Sydney, Sydney, Australia.

Correspondence: Richard J. Martin, National Jewish Health, 1400 Jackson Street, Denver, C0 80206, USA. E-mail: MartinRaNJHealth.org

@ERSpublications

A new definition of severe obstructive lung disease is needed for the biologic era. Investigators, companies and regulators must collaborate in a phenotype- and endotype-based approach to improve access to biologics for patients most likely to benefit. http://bit.ly/2Zuiakg

Cite this article as: Martin RJ, Bel EH, Pavord ID, et al. Defining severe obstructive lung disease in the biologic era: an endotype-based approach. Eur Respir J 2019; 54: 1900108 [https://doi.org/10.1183/ 13993003.00108-2019].

ABSTRACT Severe obstructive lung disease, which encompasses asthma, chronic obstructive pulmonary disease (COPD) or features of both, remains a considerable global health problem and burden on healthcare resources. However, the clinical definitions of severe asthma and COPD do not reflect the heterogeneity within these diagnoses or the potential for overlap between them, which may lead to inappropriate treatment decisions. Furthermore, most studies exclude patients with diagnoses of both asthma and COPD. Clinical definitions can influence clinical trial design and are both influenced by, and influence, regulatory indications and treatment recommendations. Therefore, to ensure its relevance in the era of targeted biologic therapies, the definition of severe obstructive lung disease must be updated so that it includes all patients who could benefit from novel treatments and for whom associated costs are justified. Here, we review evolving clinical definitions of severe obstructive lung disease and evaluate how these have influenced trial design by summarising eligibility criteria and primary outcomes of phase III randomised controlled trials of biologic therapies. Based on our findings, we discuss the advantages of a phenotype- and endotype-based approach to select appropriate populations for future trials that may influence regulatory approvals and clinical practice, allowing targeted biologic therapies to benefit a greater proportion and range of patients. This calls for co-ordinated efforts between investigators, pharmaceutical developers and regulators to ensure biologic therapies reach their full potential in the management of severe obstructive lung disease.

This article has supplementary material available from erj.ersjournals.com

Received: 15 Jan 2019 | Accepted after revision: 19 Aug 2019

Copyright $\odot$ ERS 2019 This article is open access and distributed under the terms of the Creative Commons Attribution Non-Commercial Licence 4.0. 


\section{Introduction}

Although asthma and chronic obstructive pulmonary disease (COPD) have historically been treated as overlapping syndromes $[1,2]$, the emergence of apparent mechanistic differences meant that for many years they were viewed as distinct diagnoses, with different approaches to assessment and management $[3,4]$. However, the identification of multiple phenotypes of each condition (including a subset of patients with features of both, who are often excluded from studies $[5,6])$, suggests that these diagnoses may more appropriately be viewed as a spectrum of conditions resulting from a range of pathobiological mechanisms [7]. Because the heterogeneity of these conditions is especially apparent at the severe end of the spectrum [8-10], a personalised healthcare approach based on analysis of phenotypes and underlying molecular endotypes could be particularly beneficial in patients with severe asthma and/or COPD. We use the term "severe obstructive lung disease" throughout this article to refer to patients with severe disease across both asthma and COPD diagnostic labels.

Despite continuous advancements in the diagnosis and treatment of obstructive lung disease, severe or uncontrolled asthma and COPD remain a considerable global health problem $[11,12]$. In up to $45 \%$ of patients with asthma, symptoms and/or exacerbations remain uncontrolled [13], and severe refractory asthma (persistent symptoms and exacerbations despite adherence to high-intensity treatment $[10,14]$ ) accounts for $\sim 4 \%$ of the total global asthma population of 339 million people [12, 15]. Likewise, approximately half of patients with COPD receiving "triple therapy" (inhaled corticosteroid (ICS), long-acting $\beta_{2}$-agonist (LABA) and long-acting muscarinic antagonist (LAMA)) remain symptomatic $[16,17]$ and a third continue to experience exacerbations [17]. Patients with uncontrolled severe obstructive lung disease have a substantial impact on healthcare resources [18-20]. Therefore, identifying these patients and ensuring that they receive appropriate treatment to achieve and maintain control is an important goal, particularly considering the likely high cost of novel targeted biologic therapies [21]. Several such therapies (omalizumab, mepolizumab, reslizumab, benralizumab and dupilumab) have received approval since the early 2000s for the treatment of specific subgroups of patients with severe asthma [22-30], with more in the pipeline (e.g. tezepelumab) [31, 32]. Several studies have evaluated their utility in COPD $[33,34]$. Owing to recent clinical experience and a growing body of trial data for biologic therapies, the scientific community is now in a position to reassess how severe obstructive lung disease is defined in the biologic era.

Clinical definitions and regulatory perspectives influence early-phase clinical trial design, which in turn determines later-phase trial outcomes and subsequent regulatory indications, thus affecting guideline recommendations. However, the highly restrictive eligibility criteria of randomised controlled trials (RCTs) in obstructive lung disease, including trials of biologic therapies in severe disease [35], limit their generalisability to patients in real-world clinical practice [36-42]. In this article, we aim to evaluate current definitions of severe obstructive lung disease used in clinical practice, by regulators and in clinical trials of biologic therapies, in order to inform the design of future studies and the approach to regulatory approval. We review evolving definitions of severe obstructive lung disease in relation to anti-inflammatory therapy and how these have influenced the populations included in RCTs of biologic therapies. Based on this, we provide recommendations for future research, the regulatory approach to obstructive lung disease and the use of biologics in clinical practice. We discuss an approach based on phenotypes and molecularly defined endotypes, rather than existing, nonspecific diagnostic labels, to select appropriate populations for future RCTs that may influence drug approvals and clinical practice.

\section{Current management strategies for severe obstructive lung disease}

Current management strategies for asthma and COPD commonly follow a "one-size-fits-all" approach [21], mandated by existing treatment algorithms that often recommend stepwise escalation of therapy until adequate control is achieved [43-46]. This is inconsistent with the precision medicine approach that is increasingly being called for in respiratory medicine $[5,7,21]$. Of particular concern are the indiscriminate use of high-dose ICS and the widespread reliance on oral corticosteroids (OCS) as long-term anti-inflammatory maintenance treatment in patients with persistent or refractory disease [47-49] (some of whom may also be receiving topical corticosteroid treatment for comorbidities such as nasal polyposis or atopic dermatitis $[50,51])$. Although ICS are an important component of asthma and COPD treatment strategies, guidelines recommend specialist referral and careful monitoring of patients requiring high-dose ICS (for asthma) and for patients with features of both asthma and COPD [14], and the use of blood eosinophil count combined with clinical assessment of exacerbation risk to guide ICS use (for COPD) [45]. Irreversible dose- and duration-dependent adverse effects of OCS are well documented (mostly for maintenance OCS, but with increasing evidence for effects of intermittent OCS treatment) [48, 52-55], and high-dose ICS has been associated with systemic adverse effects [56-58], including increased pneumonia risk (particularly in patients with COPD) [59,60] and clinically important local adverse effects [61]. Though ICS-induced effects may be less serious than OCS-related morbidity, they should be 
considered alongside the potential benefits of ICS treatment. The cost of future OCS-induced complications and/or treatment to prevent adverse effects $[52,53,55]$ may offset the low purchase price for payers over the long term. Recently approved and emerging biologic therapies provide effective control [31] and reduce OCS dependence in severe or uncontrolled asthma [62-64]. Evidence supports the cost-effectiveness of biologic therapies (primarily due to improvements in symptom-related quality of life, and reductions in exacerbation-related hospitalisations and asthma-related mortality risk) if carefully targeted or with substantial discounts [65].

Thus, to minimise avoidable and potentially costly adverse effects of long-term corticosteroid treatment, and to identify patients who could benefit most from alternative treatments, it is important to accurately define and diagnose severe obstructive lung disease and determine which patients are likely to respond to standard pharmacological treatments, and which may benefit from add-on biologic therapies.

\section{Clinical definitions of severe obstructive lung disease}

To summarise current clinical definitions of severe obstructive lung disease, we reviewed recent consensus and guidelines publications on severe asthma [10, 14, 21, 66, 67], severe COPD [45] and asthma-COPD overlap [14, 68-72] (summarised in table 1).

\section{Clinical definitions of severe asthma}

All five recently proposed clinical definitions of severe asthma (table 1) [10, 14, 21, 66, 67] are partly based on the level of treatment, and most specify an ICS component and at least one additional controller (LABA, OCS or other). The World Health Organization (2010) [66] and Innovative Medicine Initiative (IMI) (2011) [67] definitions required asthma to be uncontrolled (with various thresholds for symptoms and exacerbations) on high-level treatment. The IMI definition additionally included patients dependent on OCS treatment for adequate asthma control, owing to the risk of serious adverse effects with OCS treatment [67]. However, in recognition of the potential adverse effects of high-dose ICS, the definition in the more recent European Respiratory Society/American Thoracic Society (ERS/ATS) guidelines for severe asthma (2014) [10] and Global Initiative for Asthma (GINA) (2019) [14] also included dependence on high-dose ICS (for adults, equivalent to budesonide $\geqslant 1600 \mu \mathrm{g}$ per day, per ERS/ATS definition, and budesonide $>800 \mu \mathrm{g}$ per day, per GINA definition; supplementary table S1) and/or OCS for asthma control. Furthermore, GINA includes risk factors for medication side effects in its recommendation for assessing control [14].

The ERS/ATS guidelines for severe asthma recommended biologic therapy (then limited to omalizumab) for patients with severe allergic asthma [10]. These guidelines were subsequently adopted by GINA, which also recommends ICS dose escalation before considering biologic therapy [14]. Evidence shows limited or no incremental benefit at a group level for high-dose versus lower-dose ICS for improving airflow limitation, symptoms and health status in patients with asthma [73, 74], despite a significant dose-response for the frequency of oropharyngeal adverse effects [73]. This suggests that the current recommendation for escalating ICS dose in patients with severe asthma may only be effective in certain subgroups, such as those dependent on OCS [73]. The ERS/ATS guidelines highlight that there is individual variation in the dose-therapeutic efficacy of ICS [10], i.e. that limited benefit at a group level does not mean individual patients will not benefit from treatment; nevertheless, because of the risk of adverse effects, guidelines recommend only a short-term trial of high-dose ICS [14]. Otherwise, the clinical impact of adverse effects from high-dose ICS treatment [56, 57, 61] (though less severe than that of OCS-related morbidity [56]) may outweigh the limited benefit versus low-dose ICS, particularly in patients maintained on high-dose ICS in the long term.

The Lancet Commission (2018) [21] addressed the concern about ICS-related adverse effects by lowering the ICS threshold in its definition of severe asthma to "moderate dose". It stipulates that patients must have impaired lung function, variable airflow obstruction or airway eosinophilia while receiving moderate-dose ICS (with or without LABA or additional controllers, depending on the specific criterion) to be classified as having severe asthma [21]. It also includes a criterion that places greater emphasis on exacerbation risk, the rationale being that exacerbations are highly responsive to better control of lower airway inflammation with either ICS $[75,76]$ or targeted biologics [33]; thus, identifying patients at risk of exacerbations who do not respond to ICS but may respond to targeted biologics should be a priority [21]. Predictors of exacerbation risk, such as blood eosinophil count (in isolation or combination with other characteristics) [77-79], are already used to identify patients who could benefit from biologic therapies $[14,45]$. Recent evidence for alternative clinical characteristics or biomarkers that may predict treatment response independently of eosinophil count, such as nasal polyposis [80] and exhaled nitric oxide fraction (FeNO) [81, 82], highlight a need for further investigation [79]. 
Source

Asthma

\section{WHO (2010) [66] Treatment-resistant severe asthma}

- Asthma for which control is not achieved despite the highest level of recommended treatment: refractory asthma and corticosteroid-resistant asthma

- Asthma for which control can be maintained only with the highest level of recommended treatment

- "Control" is defined based on symptoms, activity limitation, night-time awakenings and SABA use in past 2-4 weeks; lung function and the number of exacerbations per year requiring OCS

\section{IMI (2011) [67] Severe refractory asthma \\ When the patient has been followed and reassessed for}

$\geqslant 6$ months:

- Uncontrolled asthma (ACQ score $\geqslant 1.5$ ) and/or

$\geqslant 2$ severe exacerbations per year despite:

$\circ$ adherence to high-dose ICS (fluticasone

$\geqslant 1000 \mu \mathrm{g}$ per day or equivalent) and/or daily OCS + LABA or another controller

o exclusion of alternative diagnoses and removal lif possiblel of sensitising substances at work/home or drugs that may cause bronchoconstriction o optimally treated comorbidities

- Asthma that can only be controlled by the use of OCS
RS/ATS (2014)

[10]

\section{Severe asthma}

- Asthma which requires treatment with guidelines-suggested medications for GINA steps 4-5 asthma (high-dose ICS and LABA or leukotriene modifier/theophyllinel for the previous year or OCS for $\geqslant 50 \%$ of the previous year to prevent it from becoming "uncontrolled" or which remains "uncontrolled" despite this therapy

- "Uncontrolled asthma" is defined as lone or more of): ACQ consistently $>1.5, A C T<20$ (or "not well controlled" by NAEPP/GINA guidelines); $\geqslant 2$ bursts of OCS ( $>3$ days each) in the previous year; $\geqslant 1$ hospitalisation, ICU stay or mechanical ventilation in the previous year; or pre-bronchodilator $\mathrm{FEV}_{1}<80 \%$ predicted and $\mathrm{FEV}_{1} / \mathrm{FVC}<\mathrm{LLN}$

- Controlled asthma that worsens on tapering of these high doses of ICS or OCS (or additional biologics)

Lancet

Commission

\section{Severe asthma}

Asthma with any of:

(2018) [21] - $\geqslant 1$ severe attack (exacerbation or flare-up)

- spirometry persistently below the normal range despite moderate-dose ICS plus one other controller

- Differentiates treatment-resistant severe asthma - Potential for inappropriate escalation of ICS from untreated or difficult-to-treat severe asthma, while recognising the importance of access to effective medications

- Excludes patients with alternative diagnoses that may mimic asthma and comorbidities that are untreated or inadequately treated and contribute to poor control

Includes patients whose asthma is controlled but dependent on high-dose ICS/OCS lencouraging step-down to assess whether asthma becomes uncontrolled)

- Provides a detailed definition of "uncontrolled". which balances symptom control with future risk

- Explicitly excludes patients who present with difficult asthma, in whom appropriate diagnosis and/or treatment of confounders le.g. poor adherence or comorbidities) "vastly improves" their current condition

- Recognises the need to reduce the risk of attacks as a priority, including addressing poor adherence and risk factors

- Avoids inappropriate escalation of ICS dose
Potential for inappropriate escalation of ICS dose

- Requires $\geqslant 2$ severe exacerbations in the previous year, exposing patients to a higher risk of OCS-related adverse effects

- Requires management of contributory factors before asthma can be classified as severe tial for inappropriate escalation of ICS dose

- A single pre-bronchodilator $\mathrm{FEV}_{1<80 \%}$ in the previous year is sufficient to categorise a patient as having uncontrolled severe asthma leven if they have had no exacerbations and have good symptom control]

- The criterion for exacerbations requires $\geqslant 2$ bursts of OCS (of $>3$ days each) in the previous year, exposing patients to a higher risk of OCS adverse effects

- Requires management of contributory factors before asthma can be classified as severe

- The first criterion may include patients with "untreated severe asthma", recognised as a separate population in the WHO definition [66], i.e. in a patient with one severe attack while 
- persistent variable airflow obstruction despite ICS/LABA

- persistent airway eosinophilia despite moderate-dose ICS

- adverse behavioural/environmental factors, including unscheduled visits, failure to attend appointments, poor adherence, smoking, allergenic environment, and the three Ds - denial, depression and disorganisation

\section{GINA (2019) [14] Severe asthma}

Asthma that requires high-dose ICS/LABA to prevent it from becoming "uncontrolled", or asthma that remains "uncontrolled" despite this treatment lafter excluding poor inhaler technique/adherence, incorrect diagnosis and comorbidities and exposure to sensitising agents/irritants)

- "Uncontrolled asthma" is defined based on symptom control and future risk of adverse outcomes, as per the GINA strategy report

\section{COPD}

\section{GOLD (2019) [45] COPD with severe airflow limitation}

Post-bronchodilator FEV $1 / F V C<0.7$ and $\mathrm{FEV}_{1}<50 \%$

predicted

- Patients are further stratified by exacerbation history and symptoms (mMRC or CAT score) using the ABCD assessment tool to guide treatment decisions

\section{Asthma-COPD overlap}

\section{Gibson and Asthma and COPD overlap syndrome}

Simpson (2009) Symptoms of increased variability of airflow and

incompletely reversible airflow limitation, including

(all of):

- Symptoms of asthma and/or COPD

- $\mathrm{FEV}_{1} / \mathrm{FVC}<0.7$

- FEV $1<80 \%$ predicted

- Airway hyper-responsiveness ${ }^{\#}$

\section{CHAIN study Asthma and COPD overlap syndrome}

(2012; 2016) COPD lage $>40$ years, with post-bronchodilator

$[69,70] \quad F E V_{1} / F V C<0.7$ and exposure to cigarette smoke) plus at

least one of:

- previous history of asthma

- bronchodilator response $>15 \%$ and $>400 \mathrm{~mL}$

OR two of:

- $\lg \mathrm{E}>100 \mathrm{IU}$

- history of atopy

- reversibility $>12 \%$ and $>200 \mathrm{~mL}$ on 2 separate visits

- blood eosinophils $>5 \%$
- Includes patients whose asthma is well controlled but dependent on high-dose ICS/OCS (encouraging step-down)

- Provides a detailed definition of "uncontrolled", which includes both symptom control and future risk

- Partly addresses heterogeneity by basing treatment decisions on exacerbations and symptoms

- Recognises the need to identify patients with features of both asthma and COPD

\section{- Based on precise diagnostic criteria}

treated only with SABA, asthma may become controlled after commencing low-dose ICS
- Requires management of contributory factors before asthma can be classified as severe

- Trial eligibility is often based on airflow limitation thresholds alone, without considering the $A B C D$ group

- Excludes other important phenotypic features such as CT scan findings and low diffusion capacity

- The term "syndrome" implies a single disease does not recognise heterogeneity within the subset of patients who meet the definition

- No recommendations for severity staging or treatment

- Excludes certain phenotypes, such as younger patients, early-onset disease and non-smokers

- The term "syndrome" implies a single disease does not recognise heterogeneity within the subset of patients who meet the definition

- No recommendations for severity staging or treatment 
TABLE 1 Continued

\begin{tabular}{|c|c|c|c|}
\hline Source & Definition & Advantages/additions to previous definitions & Disadvantages \\
\hline $\begin{array}{l}\text { Roundtable } \\
\text { consensus } \\
\text { definition (2016) } \\
\text { [71] }\end{array}$ & $\begin{array}{l}\text { Asthma-COPD overlap syndrome } \\
\text { Three major criteria, including (all of): } \\
\text { - persistent airflow limitation (FEV } 1 / F V C<0.7 \text { or } L L N) \\
\text { - } \geqslant 10 \text { pack-years' smoking history } 0 R \text { equivalent air } \\
\text { pollution exposure } \\
\text { - Documented history of asthma } 0 R \text { reversibility } \\
>400 \mathrm{~mL} \\
\text { AND at least one of: } \\
\text { - documented history of atopy or allergic rhinitis } \\
\text { - reversibility } \geqslant 200 \mathrm{~mL} \text { and } \geqslant 12 \% \text { on } \geqslant 2 \text { visits } \\
\text { - blood eosinophil count of } \geqslant 300 \text { cells per } \mu \mathrm{L}\end{array}$ & $\begin{array}{l}\text { - Provides a straightforward algorithm to facilitate } \\
\text { diagnosis and research }\end{array}$ & $\begin{array}{l}\text { - The term "syndrome" implies a single disease; } \\
\text { does not recognise heterogeneity within the } \\
\text { subset of patients who meet the definition } \\
\text { - No attempt to classify severity and limited } \\
\text { recommendations for treatment }\end{array}$ \\
\hline $\begin{array}{l}\text { GesEPOC/GEMA } \\
\text { (2017) [72] }\end{array}$ & $\begin{array}{l}\text { Asthma-COPD overlap } \\
\text { Persistent airflow limitation (FEV } 1 / F V C<0.7 \text { ) in a patient } \\
\geqslant 35 \text { years with } \geqslant 10 \text { pack-years' smoking history, who } \\
\text { does not respond to ICS/LABA and/or } 0 C S \text {, with one of: } \\
\text { - a diagnosis of current asthma (including history } \\
\text { and/or symptoms in addition to objective diagnostic } \\
\text { confirmation (reversibility } \geqslant 12 \% \text { and } \geqslant 200 \mathrm{~mL} ; \\
\text { diurnal variability in PEF } \geqslant 20 \% \text { or } F \mathrm{eNO} \geqslant 50 \mathrm{ppb} \text { )] } \\
\text { - positive bronchodilator response }(\geqslant 15 \% \text { and } \\
\geqslant 400 \mathrm{~mL} \text { ) AND/OR eosinophil count of } \geqslant 300 \text { cells per } \mu \mathrm{L}\end{array}$ & - Provides basic treatment recommendations & $\begin{array}{l}\text { - Excludes certain phenotypes, such as younger } \\
\text { patients, early-onset disease and non-smokers } \\
\text { - No attempt to classify severity; treatment is } \\
\text { based on safety considerations }\end{array}$ \\
\hline $\begin{array}{l}\text { GINA/GOLD (2019) } \\
\text { [14] }\end{array}$ & $\begin{array}{l}\text { Asthma-COPD overlap } \\
\text { Persistent airflow limitation with several features usually } \\
\text { associated with asthma and several features usually } \\
\text { associated with COPD } \\
\text { - GINA and GOLD specifically recommend against } \\
\text { attempting to define asthma-COPD overlap, because } \\
\text { of its obvious heterogeneous nature and different } \\
\text { underlying mechanisms; this is a description rather } \\
\text { than a definition } \\
\text { - A diagnosis of asthma-COPD overlap is } \\
\text { recommended if there are similar numbers of } \\
\text { features of asthma and features of COPD }\end{array}$ & $\begin{array}{l}\text { - Highlights that asthma-COPD overlap does not } \\
\text { represent a single entity } \\
\text { - Includes a wide range of potential clinical } \\
\text { phenotypes } \\
\text { - Provides basic treatment recommendations } \\
\text { based on safety }\end{array}$ & $\begin{array}{l}\text { - Characteristics, underlying mechanisms and } \\
\text { treatments for different clinical phenotypes of } \\
\text { asthma-COPD overlap are currently } \\
\text { undetermined } \\
\text { - No attempt to classify severity; treatment is } \\
\text { based on safety considerations }\end{array}$ \\
\hline
\end{tabular}

Where publications state "systemic corticosteroid", it is assumed for the purposes of this review that they refer mostly or entirely to OCS. COPD: chronic obstructive pulmonary disease; WHO: World Health Organization; IMI: Innovative Medicine Initiative; ERS: European Respiratory Society; ATS: American Thoracic Society; GINA: Global Initiative for Asthma; GOLD: Global Initiative for Obstructive Lung Disease; CHAIN: COPD History Assessment in Spain; GesEPOC: Spanish COPD Guidelines; GEMA: Spanish Guidelines on the Management of Asthma; SABA: short-acting $\beta_{2}$-agonist; OCS: oral corticosteroid; ICS: inhaled corticosteroid; ACQ: Asthma Control Questionnaire; LABA: long-acting $\beta_{2}$-agonist; ACT: Asthma Control Test; NAEPP: National Asthma Education and Prevention Program; ICU: intensive care unit; FEV1: forced expiratory volume in 1 s; FVC: forced vital capacity; LLN: lower limit of normal; mMRC: modified Medical Research Council dyspnoea scale; CAT: COPD Assessment Test; CT: computed tomography; PEF: peak expiratory flow; FeNO: exhaled nitric oxide fraction. \#: provocation dose of hypertonic saline that induces a $15 \%$ fall in $\mathrm{FEV}_{1}<12 \mathrm{~mL}$; ${ }^{9}$ : response to $400 \mu \mathrm{g}$ albuterol/salbutamol or equivalent. 
Clinical definitions of severe COPD

Unlike severe asthma, clinical gradations of COPD are not based on the required level of treatment. The Global Initiative for Chronic Obstructive Lung Disease (GOLD) 2019 report no longer defines COPD severity per se, but instead defines the severity of airflow limitation, requiring a post-bronchodilator forced expiratory volume in $1 \mathrm{~s}\left(\mathrm{FEV}_{1}\right) /$ forced vital capacity ratio of $<0.7$ as part of the definition of COPD itself, and defining airflow limitation as "severe" or "very severe" if $\mathrm{FEV}_{1}$ is <50\% predicted (table 1) [45]. Although airflow limitation thresholds often determine trial eligibility, they are not intended to guide therapy. Instead, GOLD recommends basing treatment on symptom burden and exacerbation history, with combination therapy only recommended in patients meeting specific thresholds for both or with an inadequate response to initial monotherapy [45]. Evidence for predictors of frequent COPD exacerbations, including eosinophilia [83], suggests that such predictors could be used to guide treatment decisions. This is reflected in the most recent GOLD report, which recommends using blood eosinophil count to guide ICS therapy in patients with frequent exacerbations [45]. However, other characteristics that may affect prognosis and management strategies for patients with COPD in clinical practice, such as computed tomography scan findings $[84,85]$, are not incorporated into the GOLD assessment. These characteristics may represent particular phenotypes or comorbidities of COPD that are not necessarily correlated with lung function [85], but that nevertheless should be considered alongside other assessments as part of a more personalised treatment approach. Therefore, an improved approach to identifying patients with COPD who could benefit from modified or additional treatments, regardless of spirometric severity staging, is needed. In recent RCTs, severe COPD (in terms of eligibility for biologic add-on therapy) has been defined as COPD with two or more exacerbations in the past year despite maximal inhaled therapy (i.e. triple therapy with ICS, LABA and LAMA) [33], although at present this definition is not widely used in clinical practice.

\section{Clinical definitions of severe asthma-COPD overlap}

Asthma-COPD overlap refers to the heterogeneous group of patients who have features of both asthma and COPD [14]. It does not represent a single disease [14]. To date, such patients have been excluded from pharmacotherapy RCTs, and most mechanistic studies, so this population is poorly characterised. Several groups have attempted to define asthma-COPD overlap (table 1) [14, 68-72], each proposing various algorithms incorporating the evolving clinical definitions of asthma and COPD, as well as factors that may influence treatment strategies in these patients (such as allergic status and eosinophilia). However, many of these fail to recognise the heterogeneity within this group of patients. None of the definitions propose a means of assessing severity in patients with features of both asthma and COPD. This lack of clarity highlights the need to identify underlying mechanisms associated with differential long-term clinical outcomes across the whole spectrum of obstructive lung disease. Such investigations will help to clarify which features of different phenotypic groups should be considered to represent "severe" disease. This approach may also identify biomarkers that can guide targeted therapy in a manner that is not restricted by the conflicting treatment recommendations for asthma and COPD.

Current treatment guidelines for asthma and COPD, based on studies that excluded patients with features of both, have opposite recommendations regarding the use of LABA monotherapy and ICS [6, 14, 45]. Consequently, and in the absence of evidence about underlying mechanisms, treatment recommendations for patients with features of both asthma and COPD are interim and pragmatic, based primarily on safety considerations [14]: patients with COPD who also have a diagnosis of asthma are more likely to die or be hospitalised if treated with LABA only rather than with ICS/LABA [86, 87]. Guidelines do not attempt to classify asthma-COPD overlap severity; however, similar concepts for severe asthma and severe COPD are used, in terms of persistent symptoms and/or exacerbations despite maximal inhaled therapy.

The increasing recognition of asthma-COPD overlap highlights an additional consideration around the relevance of conventional criteria for the diagnosis of asthma (variable respiratory symptoms with variable airflow limitation and reversibility [14]) and COPD (respiratory symptoms with a history of risk factors and persistent airflow limitation [45]). Studies have identified populations of patients who do not meet all of these criteria and thus have non-typical phenotypes, such as asthma with non-reversible airflow limitation [9, 88, 89] and COPD with reversible airflow limitation [90]. Therefore, in defining severe asthma and COPD it is also important to consider the criteria used to diagnose each condition, and whether a more endotype-focused approach is appropriate.

\section{Clinical trials of biologic therapies in severe obstructive lung disease}

To evaluate definitions of severe obstructive lung disease used in RCTs, we performed a PubMed search to identify publications on RCTs of biologic therapies in asthma or COPD that included the terms "severe", "moderate-to-severe", "uncontrolled" or "poorly/inadequately controlled" in the title and/or abstract (articles in English, published through to 22 May 2019; supplementary figure S1). Results were manually 
screened to identify primary publications from phase III RCTs in patients with a primary diagnosis of asthma and/or COPD.

The search returned 176 results, from which 26 relevant publications were identified, reporting trials of omalizumab [91-99], mepolizumab [33, 62, 100-102], reslizumab [103-105], benralizumab [34, 63, 106, 107], lebrikizumab [108], dupilumab [64, 109] and tralokinumab [110, 111]. Selected eligibility criteria and primary endpoints for each trial are summarised in table 2. Because only two publications reporting phase III COPD trials were identified, published phase II RCTs of biologic therapies in COPD are also discussed (summarised in supplementary table S2) [112-115].

\section{Design of existing clinical trials}

Target population and disease characteristics

In 24 of 26 publications identified, the trials had a target population of patients with severe and/or uncontrolled asthma [62-64, 91-111]. The remaining publications had target populations of patients with eosinophilic COPD (despite triple therapy) [33] or moderate-to-very severe COPD with a history of exacerbations [34]; the latter reporting two trials that failed to meet their primary endpoints of exacerbation reduction [34]. Four publications reporting phase II trials of patients with moderate-to-severe or very severe COPD were identified [112-115].

Most of the asthma trials required patients to have $\geqslant 12 \%$ bronchodilator reversibility, one of several conventional asthma diagnostic criteria commonly used when the patient is first assessed [14]. Conversely, all of the phase II and phase III COPD trials required persistent, moderate-to-severe airflow limitation as per past COPD severity staging criteria [45]. Age was also consistently used to select patients with COPD, with all of the phase II and phase III COPD trials excluding patients aged $<40$ years $(<45$ years in one trial) $[33,34,112-114]$.

All of the asthma trials had at least one criterion to select patients with uncontrolled disease, except SIRIUS [62], LIBERTY ASTHMA VENTURE [64] and TROPOS [111], which all required maintenance OCS use at entry and incorporated asthma control into the OCS dose-reduction criteria. Criteria for asthma control in RCTs have evolved: earlier trials enrolled patients based on symptom control [91, 92, 95] but more recently there has been increasing focus on the number and severity of exacerbations as inclusion criteria [33, 62, 63, 93, 94, 96-107, 110, 116] (except LAVOLTA I/II [108]). This was also the case in the phase II and phase III COPD trials, with all except the oldest study [112] having an inclusion criterion for exacerbations. Requiring a history of exacerbations as an inclusion criterion had the effect of enriching study populations for patients who were more likely to have an exacerbation during the study.

\section{Current treatment}

In line with the clinical definitions discussed above, all of the phase III trials included one or more criteria for current treatment. All of the asthma trials specified either medium- to high-dose or high-dose ICS according to GINA definitions (GINA definitions of low-, medium- and high-dose ICS are shown in supplementary table S1). The majority also specified LABA and/or additional controllers. The phase III COPD trials required either triple therapy with high-dose ICS, LABA and LAMA [33], or double or triple therapy with LABA plus LAMA and/or ICS [34]. Many asthma trials explicitly allowed OCS use in their inclusion criteria, but only SIRIUS [62], ZONDA [63], LIBERTY ASTHMA VENTURE [64] and TROPOS [111] (all designed to evaluate OCS sparing) mandated it. Eight asthma studies excluded patients with chronic or maintenance OCS use at baseline, either at all or at various dose thresholds [92-95, 104, $105,108,110]$.

\section{Phenotype}

Most of the trials were restricted to a specific phenotype appropriate to the molecular target of the treatment. Thus, all trials of omalizumab (anti-IgE) only enrolled patients with evidence of IgE-mediated allergic asthma [91-99], whereas trials of mepolizumab or reslizumab (anti-interleukin (IL)-5) or benralizumab (anti-IL-5 receptor) enrolled or performed primary analyses on patients with sputum or blood eosinophil counts above a specific threshold [33,34,62,63, 100-104, 106, 107, 113] (with the exception of Corren et al. [105]). Only the DREAM trial of mepolizumab (a goal of which was to identify characteristics, including biomarkers, that predicted response) had an inclusion criterion for FeNO [100]. The LAVOLTA trials of lebrikizumab (anti-IL-13) performed primary analyses on patients with a "biomarker-high" phenotype of higher concentrations of the Type 2 (T2) inflammatory marker periostin and/or blood eosinophilia [108]. The two trials of dupilumab (which blocks IL-4 and IL-13 signalling via the IL-4 receptor) did not restrict eligibility based on T2 inflammatory markers [64, 109]. The STRATOS 2 trial of tralokinumab (anti-IL-13) specified a primary analysis population of patients with $F$ eNO $\geqslant 37 \mathrm{ppb}$, which was identified as the preferred "biomarker-high" population in the all-comers trial, STRATOS 1 [110]. 


\begin{tabular}{|c|c|c|c|c|c|c|c|c|c|}
\hline Trial (treatment) & $\begin{array}{l}\text { Authors' description of } \\
\text { target population }\end{array}$ & Severity/control & Current treatment & $\begin{array}{l}\text { Bronchodilator } \\
\text { reversibility } \\
\text { requirement (see } \\
\text { footnotes) }\end{array}$ & Eosinophilic status & Feno requirement & $\begin{array}{l}\text { Allergy/atopy } \\
\text { requirement }\end{array}$ & Notable exclusions & Primary endpoint \\
\hline \multicolumn{10}{|c|}{ Target population: patients with asthma } \\
\hline $\begin{array}{r}\text { Busse et al. [91] } \\
\text { (omalizumab) }\end{array}$ & Severe allergic asthma & $\begin{array}{l}\text { Symptomatic (total } \\
\text { daily symptom } \\
\text { score } \geqslant 3 \text { ) }\end{array}$ & $\begin{array}{l}\text { ICS } 420-840 \mu \mathrm{g} \text { per } \\
\text { day (BDP or } \\
\text { equivalent) }{ }^{\#}\end{array}$ & $\geqslant 12 \%$ & n/a & $\mathrm{n} / \mathrm{a}$ & $\begin{array}{l}\text { Positive skin-prick, } \\
\text { IgE } 30-700 \text { IU.mL }{ }^{-1}\end{array}$ & $\mathrm{n} / \mathrm{a}$ & $\begin{array}{l}\text { Number of } \\
\text { exacerbations } \\
\text { (during ICS stable } \\
\text { and ICS reduction } \\
\text { phases) }\end{array}$ \\
\hline $\begin{array}{l}\text { Solèr et al. [92] } \\
\text { (omalizumab) }\end{array}$ & Allergic asthma & $\begin{array}{l}\text { Symptomatic (total } \\
\text { daily symptom } \\
\text { score } \geqslant 3 \text { ) }\end{array}$ & $\begin{array}{l}\text { Medium- to high-dose } \\
\text { ICS (BDP 500- } \\
1200 \mu \mathrm{\mu} \text { per day or } \\
\text { equivalent) } \\
+\boldsymbol{\beta}_{2} \text {-agonist as } \\
\text { needed for } \\
\text { maintenance }\end{array}$ & $\geqslant 12 \%$ & $\mathrm{n} / \mathrm{a}$ & $\mathrm{n} / \mathrm{a}$ & $\begin{array}{l}\text { Positive skin-prick, } \\
\text { IgE } 30-700 \mathrm{IU} \cdot \mathrm{mL}^{-1}\end{array}$ & Maintenance OCS use & $\begin{array}{l}\text { Number of } \\
\text { exacerbations } \\
\text { (during ICS stable } \\
\text { and ICS reduction } \\
\text { phases) }\end{array}$ \\
\hline $\begin{array}{l}\text { SOLAR [93] } \\
\quad \text { (omalizumab) }\end{array}$ & $\begin{array}{c}\text { Concomitant allergic } \\
\text { asthma and PAR }\end{array}$ & $\begin{array}{l}\text { Concomitant } \\
\text { moderate-to-severe } \\
\text { PAR } \geqslant 2 \text { years; } \\
\text { AQLQ total score } \\
>64 \text { and RQLQ total } \\
\text { score }>54 ; \geqslant 2 \\
\text { unscheduled visits } \\
\text { for asthma in the } \\
\text { past year or } \geqslant 3 \text { in } \\
\text { past } 2 \text { years }\end{array}$ & $\begin{array}{l}\text { Medium- to high-dose } \\
\text { ICS (budesonide } \\
\geqslant 400 \mu \mathrm{g} \mathrm{per} \mathrm{day)}\end{array}$ & $\geqslant 12 \%$ & n/a & $\mathrm{n} / \mathrm{a}$ & $\begin{array}{l}\text { Positive skin-prick, } \\
\text { IgE 30-1300 IU.mL }{ }^{-1}\end{array}$ & OCS use & $\begin{array}{l}\text { (Co-primary) Number } \\
\text { of exacerbations and } \\
\text { proportion of } \\
\text { patients with } \\
\text { improvement in both } \\
\text { asthma and rhinitis } \\
\text { quality-of-life scores }\end{array}$ \\
\hline $\begin{array}{l}\text { INNOVATE [94] } \\
\text { (omalizumab) }\end{array}$ & Severe persistent asthma & $\begin{array}{l}\text { Daytime or night-time } \\
\text { symptoms; } \geqslant 2 \\
\text { exacerbations } \\
\text { requiring OCS in } \\
\text { the past year or } \geqslant 1 \\
\text { severe exacerbation } \\
\text { requiring } \\
\text { hospitalisation or } \\
\text { ER treatment in the } \\
\text { past year }\end{array}$ & 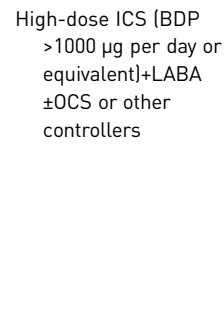 & $\geqslant 12 \%$ & $\mathrm{n} / \mathrm{a}$ & n/a & $\begin{array}{l}\text { Positive skin-prick, } \\
\text { IgE } 30-700 \mathrm{IU} \cdot \mathrm{mL}^{-1}\end{array}$ & $\begin{array}{l}\text { Maintenance OCS use } \\
>20 \mathrm{mg} \text { per day, } \\
1 \leqslant 20 \mathrm{mg} \text { per day } \\
\text { was permitted } \\
\text { providing } \geqslant 1 \\
\text { exacerbation in the } \\
\text { past year occurred } \\
\text { on this therapy); } \\
\text { smokers or former } \\
\text { smokers with } \\
\geqslant 10 \text { pack-years }\end{array}$ & $\begin{array}{l}\text { Rate of clinically } \\
\text { significant asthma } \\
\text { exacerbations }\end{array}$ \\
\hline $\begin{array}{l}\text { Ohta et al. [95] } \\
\text { (omalizumab) } \\
\text { NCT00232050 }\end{array}$ & $\begin{array}{l}\text { Moderate-to-severe } \\
\text { persistent asthma }\end{array}$ & $\begin{array}{l}\text { Moderate-to-severe } \\
\text { asthma as per } \\
\text { GINA 2002; daytime } \\
\text { and/or night-time } \\
\text { symptoms }\end{array}$ & $\begin{array}{l}\text { Medium- to high-dose } \\
\text { ICS (BDP } \geqslant 800 \mu \mathrm{g} \\
\text { per day or } \\
\text { equivalent)+ } \\
\geqslant 1 \text { LABA, OCS or } \\
\text { other controllers }\end{array}$ & $\mathrm{n} / \mathrm{a}$ & $\mathrm{n} / \mathrm{a}$ & $\mathrm{n} / \mathrm{a}$ & $\begin{array}{l}\text { Positive skin-prick or } \\
\text { in vitro reactivity, } \\
\text { IgE } 30-700 \mathrm{IU} \cdot \mathrm{mL}^{-1}\end{array}$ & $\begin{array}{l}\text { Maintenance OCS use } \\
\text { (>10 mg.day); } \\
\text { complicated } \\
\text { pulmonary disease } \\
\text { considered to } \\
\text { interfere with } \\
\text { evaluation }\end{array}$ & $\begin{array}{l}\text { Change from baseline } \\
\text { in morning PEF }\end{array}$ \\
\hline $\begin{array}{r}\text { Lanier et al. [96] } \\
\text { (omalizumab) } \\
\text { NCT00079937 }\end{array}$ & $\begin{array}{l}\text { Moderate-to-severe, } \\
\text { uncontrolled allergic } \\
\text { asthma (children) }\end{array}$ & $\begin{array}{l}\text { Daytime or night-time } \\
\text { symptoms } ; \geqslant 2 \\
\text { exacerbations in } \\
\text { the past year or } \geqslant 3 \\
\text { in past } 2 \text { years or } \\
\geqslant 1 \text { severe } \\
\text { exacerbation } \\
\text { requiring } \\
\text { hospitalisation in } \\
\text { the past year }\end{array}$ & $\begin{array}{l}\text { Medium- to high-dose } \\
\text { ICS (FP } \geqslant 200 \mu g \\
\text { per day or } \\
\text { equivalent) }\end{array}$ & $\geqslant 12 \%$ & $\mathrm{n} / \mathrm{a}$ & $\mathrm{n} / \mathrm{a}$ & $\begin{array}{l}\text { Positive skin-prick, } \\
\text { IgE 30-1300 IU.mL }\end{array}$ & $\begin{array}{l}\text { OCS use for reasons } \\
\text { other than asthma }\end{array}$ & $\begin{array}{l}\text { Rate of clinically } \\
\text { significant asthma } \\
\text { exacerbations }\end{array}$ \\
\hline
\end{tabular}




\begin{tabular}{|c|c|c|c|c|c|c|c|c|c|}
\hline Trial (treatment) & $\begin{array}{l}\text { Authors' description of } \\
\text { target population }\end{array}$ & Severity/control & Current treatment & $\begin{array}{l}\text { Bronchodilator } \\
\text { reversibility } \\
\text { requirement (see } \\
\text { footnotes) }\end{array}$ & Eosinophilic status & Feno requirement & $\begin{array}{l}\text { Allergy/atopy } \\
\text { requirement }\end{array}$ & Notable exclusions & Primary endpoint \\
\hline $\begin{array}{l}\text { EXTRA [97] } \\
\text { (omalizumab) } \\
\text { NCT00314574 }\end{array}$ & $\begin{array}{l}\text { Severe, uncontrolled } \\
\text { allergic asthma }\end{array}$ & $\begin{array}{l}\text { Daytime and } \\
\text { night-time } \\
\text { symptoms } \\
\text { requiring SABA; } \geqslant 1 \\
\text { exacerbation in the } \\
\text { past year }\end{array}$ & $\begin{array}{l}\text { High-dose ICS } \\
\text { (fluticasone } \\
\geqslant 500 \mu \mathrm{g} \text { twice daily } \\
\text { or equivalent) } \\
\quad+\text { LABA } \pm \text { OCS or } \\
\text { other controllers }\end{array}$ & $\mathrm{n} / \mathrm{a}$ & $\mathrm{n} / \mathrm{a}$ & $n / a$ & $\begin{array}{l}\text { Positive skin-prick or } \\
\text { in vitro reactivity, } \\
\text { IgE } 30-700 \mathrm{IU} \cdot \mathrm{mL}^{-1}\end{array}$ & $\begin{array}{l}\text { Exacerbation requiring } \\
\text { OCS or increase in } \\
\text { baseline OCS in } \\
\leqslant 30 \text { days prior to } \\
\text { screening; smokers } \\
\text { or former smokers } \\
\text { with } \geqslant 10 \\
\text { pack-years; active } \\
\text { lung disease other } \\
\text { than asthma }\end{array}$ & Rate of exacerbations \\
\hline $\begin{array}{r}\text { Garcia et al. [98] } \\
\text { (omalizumab) } \\
\text { NCT01007149 }\end{array}$ & $\begin{array}{l}\text { Severe, persistent, } \\
\text { uncontrolled, } \\
\text { non-atopic asthma }\end{array}$ & $\begin{array}{l}\text { Severe uncontrolled } \\
\text { asthma as per } \\
\text { GINA 2006; } \geqslant 2 \\
\text { exacerbations per } \\
\text { year and/or } \geqslant 1 \\
\text { exacerbation } \\
\text { requiring } \\
\text { hospitalisation or } \\
\text { ER treatment in the } \\
\text { past year }\end{array}$ & $\begin{array}{l}\text { High-dose ICS (BDP } \\
\quad>1000 \mu \text { per day } \\
\text { or equivalent) } \\
\quad+\text { LABA } \pm \text { OCS }\end{array}$ & $n / a$ & $n / a$ & $n / a$ & $\begin{array}{l}\text { Negative Phadiatop, } \\
\text { radioallergosorbent } \\
\text { and skin-prick tests; } \\
\text { lgE } 30-700 \mathrm{IU} \cdot \mathrm{mL}^{-1}\end{array}$ & $\begin{array}{l}\text { Smokers or former } \\
\text { smokers with } \geqslant 10 \\
\text { pack-years; } \\
\text { uncontrolled other } \\
\text { chronic diseases }\end{array}$ & $\begin{array}{l}\text { Change from baseline } \\
\text { in cell surface } \\
\text { high-affinity IgE } \\
\text { receptor (FcRRI) } \\
\text { expression on } \\
\text { basophils and } \\
\text { plasmacytoid } \\
\text { dendritic cells }\end{array}$ \\
\hline $\begin{array}{l}\text { Li et al. [99] } \\
\quad \text { lomalizumab] } \\
\text { NCT01202903 }\end{array}$ & $\begin{array}{l}\text { Moderate-to-severe } \\
\text { allergic asthma }\end{array}$ & $\begin{array}{l}\text { Moderate-to-severe } \\
\text { asthma as per } \\
\text { GINA 2014; } \geqslant 2 \\
\text { exacerbations in } \\
\text { the past year or } \geqslant 3 \\
\text { in past } 2 \text { years }\end{array}$ & $\begin{array}{l}\text { Medium- to high-dose } \\
\text { ICS (per GINA } \\
2014 \text { )+LABA }\end{array}$ & $\geqslant 12 \%$ & $n / a$ & $n / a$ & $\begin{array}{l}\text { A positive reaction to } \\
\geqslant 1 \text { perennial } \\
\text { aeroallergen, } \\
\text { IgE } 30-700 \text { IU } \cdot \mathrm{mL}^{-1}\end{array}$ & $\begin{array}{l}\text { Active lung disease } \\
\text { other than allergic } \\
\text { asthma }\end{array}$ & $\begin{array}{l}\text { Mean change from } \\
\text { baseline in morning } \\
\text { PEF }\end{array}$ \\
\hline $\begin{array}{l}\text { DREAM [100] } \\
\text { (mepolizumab) } \\
\text { NCT01000506 }\end{array}$ & $\begin{array}{l}\text { Severe eosinophilic } \\
\text { asthma }\end{array}$ & $\begin{array}{l}\text { Refractory asthma per } \\
\text { ERS/ATS definition; } \\
\text { one of } \geqslant 2 \\
\text { exacerbations in } \\
\text { the past year, } \\
\text { prompt } \\
\text { deterioration of } \\
\text { asthma control } \\
\text { after } \leqslant 25 \% \\
\text { reduction in } \\
\text { maintenance ICS or } \\
\text { OCS, eosinophilia, } \\
\text { or elevated Feno }\end{array}$ & $\begin{array}{l}\text { High-dose ICS (FP } \\
\geqslant 880 \mu \mathrm{\mu g} \text { per day or } \\
\text { equivalent) } \pm \text { OCS } \\
\text { +additional } \\
\text { controllers }\end{array}$ & $>12 \%$ and $200 \mathrm{~mL}$ & $\begin{array}{l}\text { Any indicator of } \\
\text { eosinophilic } \\
\text { inflammation, } \\
\text { including sputum } \\
\text { eosinophil count of } \\
\geqslant 3 \% \text { or } \\
\text { asthma-related } \\
\text { blood eosinophil } \\
\text { count of } \\
\geqslant 300 \text { cells per } \mu \mathrm{L}\end{array}$ & $\begin{array}{l}\text { Any indicator of } \\
\text { eosinophilic } \\
\text { inflammation, } \\
\text { including } \\
F_{\mathrm{eNO} O 50 \mathrm{ppb}}\end{array}$ & $\begin{array}{l}\text { Positive } \\
\text { radioallergosorbent } \\
\text { test }\end{array}$ & $\begin{array}{l}\text { Smokers or former } \\
\text { smokers with } \geqslant 10 \\
\text { pack-years; } \\
\text { substantial } \\
\text { uncontrolled } \\
\text { comorbidity }\end{array}$ & $\begin{array}{l}\text { Rate of clinically } \\
\text { significant asthma } \\
\text { exacerbations }\end{array}$ \\
\hline $\begin{array}{l}\text { MENSA [101] } \\
\text { (mepolizumab) } \\
\text { NCT01691521 }\end{array}$ & $\begin{array}{l}\text { Severe eosinophilic } \\
\text { asthma }\end{array}$ & $\begin{array}{l}\geqslant 2 \text { exacerbations } \\
\text { requiring OCS or } \\
\geqslant 2 \text {-fold increase in } \\
\text { usual OCS dose in } \\
\text { the past year }\end{array}$ & $\begin{array}{l}\text { High-dose ICS (FP } \\
\geqslant 880 \mu \mathrm{pg} \text { per day or } \\
\text { equivalent)+an } \\
\text { additional } \\
\text { controller }\end{array}$ & $\geqslant 12 \% \pi$ & $\begin{array}{l}\text { Blood eosinophil } \\
\text { count of } \geqslant 300 \text { cells } \\
\text { per } \mu \mathrm{L} \text { during the } \\
\text { previous year or of } \\
\geqslant 150 \text { cells per } \mu \mathrm{L} \\
\text { during the } \\
\text { optimisation phase }\end{array}$ & n/a & $n / a$ & $\begin{array}{l}\text { Smokers or former } \\
\text { smokers with } \geqslant 10 \\
\text { pack-years; } \\
\text { clinically important } \\
\text { lung condition other } \\
\text { than asthma } \\
\text { (including COPD) }\end{array}$ & $\begin{array}{l}\text { Rate of clinically } \\
\text { significant asthma } \\
\text { exacerbations }\end{array}$ \\
\hline
\end{tabular}




\begin{tabular}{|c|c|c|c|c|c|c|c|c|c|}
\hline Trial (treatment) & $\begin{array}{l}\text { Authors' description of } \\
\text { target population }\end{array}$ & Severity/control & Current treatment & $\begin{array}{l}\text { Bronchodilator } \\
\text { reversibility } \\
\text { requirement (see } \\
\text { footnotes) }\end{array}$ & Eosinophilic status & Feno requirement & $\begin{array}{l}\text { Allergy/atopy } \\
\text { requirement }\end{array}$ & Notable exclusions & Primary endpoint \\
\hline $\begin{array}{l}\text { SIRIUS [62] } \\
\quad \text { (mepolizumab) } \\
\text { NCT01691508 }\end{array}$ & $\begin{array}{l}\text { Severe eosinophilic } \\
\text { asthma }\end{array}$ & n/a & $\begin{array}{l}\text { High-dose ICS (FP } \\
\geqslant 880 \mu \mathrm{\mu g} \text { per day or } \\
\text { equivalentl)+an } \\
\text { additional } \\
\text { controller (for } \\
\geqslant 3 \text { months in } \\
\text { previous } \\
12 \text { months)+OCS } \\
\text { (equivalent to } \\
\text { prednisone 5- } \\
35 \mathrm{mg} \text { per day, for } \\
\text { past } 6 \text { months) }\end{array}$ & $\geqslant 12 \%$ and $200 \mathrm{~mL}^{+}$ & $\begin{array}{l}\text { Blood eosinophil } \\
\text { count of } \geqslant 300 \text { cells } \\
\text { per } \mu L \text { within } \\
1 \text { year of screening } \\
\text { or of } \geqslant 150 \text { cells } \\
\text { per } \mu \mathrm{L} \text { at screening }\end{array}$ & n/a & $n / a$ & $\begin{array}{l}\text { Smokers or former } \\
\text { smokers with } \geqslant 10 \\
\text { pack-years; } \\
\text { clinically important } \\
\text { lung condition other } \\
\text { than asthma } \\
\text { (including COPD) }\end{array}$ & $\begin{array}{l}\% \text { reduction in daily } \\
\text { OCS dose from } \\
\text { optimised dose to } \\
\text { weeks } 20-24^{8}\end{array}$ \\
\hline $\begin{array}{l}\text { MUSCA [102] } \\
\text { (mepolizumab) } \\
\text { NCT02281318 }\end{array}$ & $\begin{array}{l}\text { Severe eosinophilic } \\
\text { asthma }\end{array}$ & $\begin{array}{l}\text { Severe uncontrolled } \\
\text { asthma per ERS/ } \\
\text { ATS definition; } \geqslant 2 \\
\text { exacerbations } \\
\text { requiring OCS or } \\
\geqslant 2 \times \text { increase in } \\
\text { usual OCS dose in } \\
\text { the past year }\end{array}$ & $\begin{array}{l}\text { High-dose ICS+ } \\
\geqslant 1 \text { additional } \\
\text { controller }\end{array}$ & $n / a$ & $\begin{array}{l}\text { Blood eosinophil } \\
\text { count of } \geqslant 300 \text { cells } \\
\text { per } \mu L \text { within } \\
1 \text { year of screening } \\
\text { or of } \geqslant 150 \text { cells } \\
\text { per } \mu L \text { at screening }\end{array}$ & n/a & $n / a$ & $\begin{array}{l}\text { Smokers or former } \\
\text { smokers with } \geqslant 10 \\
\text { pack-years; } \\
\text { concurrent } \\
\text { respiratory disease }\end{array}$ & $\begin{array}{l}\text { Mean change from } \\
\text { baseline in SGRQ } \\
\text { total score }\end{array}$ \\
\hline $\begin{array}{l}\text { Castro et al. Study 1, } \\
\text { Study } 2 \text { [103] } \\
\text { (reslizumab) } \\
\text { NCT01287039, } \\
\text { NCT01285323 }\end{array}$ & $\begin{array}{l}\text { Inadequately controlled, } \\
\text { moderate-to-severe } \\
\text { eosinophilic asthma }\end{array}$ & $\begin{array}{l}\text { ACQ-7 score } \geqslant 1.5 \\
\geqslant 1 \text { exacerbation } \\
\text { requiring OCS in } \\
\text { the past year }\end{array}$ & $\begin{array}{l}\text { Medium- to high-dose } \\
\text { ICS (FP } \geqslant 440 \mu \mathrm{g} \\
\text { per day or } \\
\text { equivalent) } \\
\text { additional } \\
\text { controller } \\
\text { (including OCS) }\end{array}$ & $\geqslant 12 \%$ & $\begin{array}{l}\text { Blood eosinophil } \\
\text { count of } \geqslant 400 \text { cells } \\
\text { per } \mu \mathrm{L}\end{array}$ & $n / a$ & $\mathrm{n} / \mathrm{a}$ & $\begin{array}{l}\text { Current smokers; } \\
\text { another confounding } \\
\text { underlying lung } \\
\text { disorder lincluding } \\
\text { COPDI }\end{array}$ & $\begin{array}{l}\text { Rate of clinically } \\
\text { significant asthma } \\
\text { exacerbations }\end{array}$ \\
\hline $\begin{array}{l}\text { Bjermer et al. [104] } \\
\text { (reslizumab) } \\
\text { NCT01270464 }\end{array}$ & $\begin{array}{l}\text { Inadequately controlled } \\
\text { asthma with elevated } \\
\text { blood eosinophils }\end{array}$ & ACQ-7 score $\geqslant 1.5$ & $\begin{array}{l}\text { Medium- to high-dose } \\
\text { ICS (FP } \\
\geqslant 440 \mu \mathrm{g} \text { per day or } \\
\text { equivalent) } \pm \text { an } \\
\text { additional } \\
\text { controller }\end{array}$ & $\geqslant 12 \%$ & $\begin{array}{l}\text { Blood eosinophil } \\
\text { count of } \geqslant 400 \text { cells } \\
\text { per } \mu \mathrm{L}\end{array}$ & $\mathrm{n} / \mathrm{a}$ & $n / a$ & $\begin{array}{l}\text { Maintenance OCS use; } \\
\text { current smokers; } \\
\text { other confounding } \\
\text { lung disorders or } \\
\text { pulmonary } \\
\text { conditions }\end{array}$ & $\begin{array}{l}\text { Change from } \\
\text { baseline in pre- } \\
\text { bronchodilator } \mathrm{FEV}_{1}\end{array}$ \\
\hline $\begin{array}{l}\text { Corren et al. [105] } \\
\quad \text { (reslizumab) } \\
\text { NCT01508936 }\end{array}$ & Poorly controlled asthma & ACQ-7 score $\geqslant 1.5$ & $\begin{array}{l}\text { Medium- to high-dose } \\
\text { ICS (FP } \\
\geqslant 440 \mu \mathrm{gg} \cdot \text { day or } \\
\text { equivalentltan } \\
\text { additional } \\
\text { controller }\end{array}$ & $\geqslant 12 \%$ & $n / a$ & $\mathrm{n} / \mathrm{a}$ & $\mathrm{n} / \mathrm{a}$ & $\begin{array}{l}\text { Maintenance OCS use; } \\
\text { current smokers; } \\
\text { underlying lung } \\
\text { disorders or } \\
\text { pulmonary } \\
\text { conditions }\end{array}$ & $\begin{array}{l}\text { Change from baseline } \\
\text { in } \mathrm{FEV}_{1}\end{array}$ \\
\hline $\begin{array}{l}\text { CALIMA [106] } \\
\text { (benralizumab) } \\
\text { NCT01914757 }\end{array}$ & $\begin{array}{l}\text { Severe, uncontrolled } \\
\text { eosinophilic asthma }\end{array}$ & $\begin{array}{l}\text { ACQ-6 score } \geqslant 1.5 ; \\
\geqslant 2 \text { exacerbations } \\
\text { requiring OCS or } \\
\text { increase in usual } \\
\text { OCS dose in the } \\
\text { past year }\end{array}$ & $\begin{array}{l}\text { High-dose ICS (FP } \\
\geqslant 500 \mu \mathrm{g} \text { per day or } \\
\text { equivalent)+LABA } \\
\pm 0 C S \text { and } \\
\text { additional } \\
\text { controllers }\end{array}$ & $\geqslant 12 \%$ and $200 \mathrm{~mL}$ & $\begin{array}{l}\text { Blood eosinophil } \\
\text { count of }<300 \text { cells } \\
\text { per } \mu \mathrm{L} \text { or of } \\
\geqslant 300 \text { cells per } \mu \mathrm{L} \\
(\geqslant 300 \text { cells per } \mu \mathrm{L} \\
\text { in primary analysis } \\
\text { population) }\end{array}$ & $\mathrm{n} / \mathrm{a}$ & $\mathrm{n} / \mathrm{a}$ & $\begin{array}{l}\text { Clinically important } \\
\text { pulmonary or } \\
\text { eosinophilic disease } \\
\text { other than asthma } \\
\text { (including COPD) }\end{array}$ & $\begin{array}{l}\text { AER ratio versus } \\
\text { placebo for patients } \\
\text { receiving fluticasone } \\
\geqslant 500 \mu \mathrm{g} \text { or } \\
\text { equivalent plus } \\
\text { LABA with baseline } \\
\text { blood eosinophils } \\
\geqslant 300 \text { cells per } \mu \mathrm{L}\end{array}$ \\
\hline
\end{tabular}




\begin{tabular}{|c|c|c|c|c|c|c|c|c|c|}
\hline Trial (treatment) & $\begin{array}{l}\text { Authors' description of } \\
\text { target population }\end{array}$ & Severity/control & Current treatment & $\begin{array}{l}\text { Bronchodilator } \\
\quad \text { reversibility } \\
\text { requirement (see } \\
\text { footnotes) }\end{array}$ & Eosinophilic status & Feno requirement & $\begin{array}{l}\text { Allergy/atopy } \\
\text { requirement }\end{array}$ & Notable exclusions & Primary endpoint \\
\hline $\begin{array}{l}\text { SIROCCO [107] } \\
\text { (benralizumab) } \\
\text { NCT01928771 }\end{array}$ & $\begin{array}{l}\text { Severe, uncontrolled } \\
\text { eosinophilic asthma }\end{array}$ & $\begin{array}{l}\text { ACQ-6 score } \geqslant 1 ; \\
\geqslant 2 \text { exacerbations } \\
\text { requiring OCS or } \\
\text { increase in usual } \\
\text { OCS dose in the } \\
\text { past year }\end{array}$ & $\begin{array}{l}\text { High-dose ICS (FP } \\
\geqslant 500 \mu \mathrm{g} \text { per day or } \\
\text { equivalent)+LABA } \\
\pm 0 C S \text { and } \\
\text { additional } \\
\text { controller }\end{array}$ & $\geqslant 12 \%$ and $200 \mathrm{~mL}$ & $\begin{array}{l}\text { Blood eosinophil } \\
\text { count of }<300 \text { cells } \\
\text { per } \mu \mathrm{L} \text { or of } \\
\geqslant 300 \text { cells per } \mu \mathrm{L} \\
\text { ( } \geqslant 300 \text { cells per } \mu \mathrm{L} \\
\text { in primary analysis } \\
\text { population) }\end{array}$ & $\mathrm{n} / \mathrm{a}$ & $n / a$ & $\begin{array}{l}\text { Clinically important } \\
\text { pulmonary or } \\
\text { eosinophilic disease } \\
\text { other than asthma } \\
\text { (including COPD) }\end{array}$ & $\begin{array}{l}\text { AER ratio versus } \\
\text { placebo for patients } \\
\text { with baseline blood } \\
\text { eosinophils } \\
\geqslant 300 \text { cells per } \mu \mathrm{L}\end{array}$ \\
\hline $\begin{array}{l}\text { ZONDA [63] } \\
\text { (benralizumab) } \\
\text { NCT02075255 }\end{array}$ & $\begin{array}{l}\text { Severe eosinophilic } \\
\text { asthma requiring OCS }\end{array}$ & $\begin{array}{l}\geqslant 1 \text { exacerbations in } \\
\text { the past year }\end{array}$ & $\begin{array}{l}\text { High-dose ICS } \\
\text { (fluticasone } \\
>500 \mu \mathrm{g} \text { per day or } \\
\text { equivalent)+LABA } \\
\text { +OCS lequivalent to } \\
\text { prednisone } 7.5- \\
40 \mathrm{mg} \text { per day, for } \\
\text { past } 6 \text { months) }\end{array}$ & $\begin{array}{l}\geqslant 12 \% \text { and } 200 \mathrm{~mL} \mathrm{~L}^{\pi} \text { or } \\
\text { documented } \\
\text { reversibility during } \\
\text { past } 2 \text { years }\end{array}$ & $\begin{array}{l}\text { Blood eosinophil } \\
\quad \text { count of } \\
\geqslant 150 \text { cells per } \mu \mathrm{L}\end{array}$ & $\mathrm{n} / \mathrm{a}$ & n/a & $\begin{array}{l}\text { Clinically important } \\
\text { pulmonary or } \\
\text { eosinophilic disease } \\
\text { other than asthma } \\
\text { (including COPD) }\end{array}$ & $\begin{array}{l}\% \text { reduction in daily } \\
\text { OCS dose from } \\
\text { baseline to end of } \\
\text { maintenance phase } \\
\text { while maintaining } \\
\text { asthma control }{ }^{f}\end{array}$ \\
\hline $\begin{array}{l}\text { LAVOLTA I, } \\
\text { LAVOLTA II [108] } \\
\text { (lebrikizumab) } \\
\text { NCT01867125, } \\
\text { NCT01868061 }\end{array}$ & Uncontrolled asthma & $\begin{array}{l}\text { ACQ- } 5 \text { score } \geqslant 1.5 ; \text { at } \\
\text { least one of } \\
\text { symptoms } \geqslant 2 \text { days/ } \\
\text { week, night-time } \\
\text { awakenings } \\
\geqslant 1 \text { night per week, } \\
\text { SABA } \geqslant 2 \text { days per } \\
\text { week or } \\
\text { interference with } \\
\text { daily activities }\end{array}$ & $\begin{array}{l}\text { High-dose ICS (FP } \\
500-2000 \mu \mathrm{g} \text { per } \\
\text { day or equivalent) } \\
\quad+\geqslant 1 \text { additional } \\
\text { controller }\end{array}$ & $\geqslant 12 \%$ & $\begin{array}{l}\text { Blood eosinophil } \\
\text { count of }<300 \text { cells } \\
\text { per } \mu \mathrm{L} \text { or of } \\
\geqslant 300 \text { cells per } \mu \mathrm{L} \\
(\geqslant 300 \text { cells per } \mu \mathrm{L} \\
\text { and/or periostin } \\
\geqslant 50 \mathrm{ng} \cdot \mathrm{mL}^{-1} \text { in } \\
\text { primary analysis } \\
\text { population) }\end{array}$ & $\mathrm{n} / \mathrm{a}$ & n/a & $\begin{array}{l}\text { Maintenance OCS use } \\
\text { within past } \\
3 \text { months; smokers } \\
\text { or former smokers } \\
\text { with } \geqslant 10 \\
\text { pack-years; } \\
\text { clinically significant } \\
\text { lung disease other } \\
\text { than asthma }\end{array}$ & $\begin{array}{l}\text { AER in "biomarker- } \\
\text { high" patients } \\
\text { (periostin } \\
\geqslant 50 \mathrm{ng} \cdot \mathrm{mL} \text { and/or } \\
\text { blood eosinophils } \\
\geqslant 300 \text { cells per } \mu \mathrm{L} \text { ) }\end{array}$ \\
\hline $\begin{array}{l}\text { LIBERTY ASTHMA } \\
\text { QUEST [109] } \\
\text { (dupilumab) } \\
\text { NCT02414854 }\end{array}$ & $\begin{array}{l}\text { Moderate-to-severe, } \\
\text { uncontrolled asthma }\end{array}$ & $\begin{array}{l}\text { ACQ }-5 \text { score } \geqslant 1.5 ; \geqslant 1 \\
\text { exacerbation in past } \\
\text { year requiring } \\
\text { hospitalisation, } \\
\text { emergency medical } \\
\text { care or OCS for } \\
\geqslant 3 \text { days }\end{array}$ & $\begin{array}{l}\text { High-dose ICS (FP } \\
\geqslant 500 \mu \mathrm{g} \text { per day or } \\
\text { equivalent)+up to } 2 \\
\text { additional } \\
\text { controllers }\end{array}$ & $\geqslant 12 \%$ and $200 \mathrm{~mL}$ & $n / a$ & n/a & n/a & $\begin{array}{l}\text { Current smokers, or } \\
\text { former smokers } \\
\text { with >10 pack-years; } \\
\text { COPD or other lung } \\
\text { disease that may } \\
\text { impair lung function }\end{array}$ & $\begin{array}{l}\text { (Co-primary) Severe } \\
\text { AER and change } \\
\text { from baseline in } \\
\text { pre-bronchodilator } \\
\text { FEV }_{1}\end{array}$ \\
\hline $\begin{array}{l}\text { LIBERTY ASTHMA } \\
\text { VENTURE [64] } \\
\text { (dupilumab) } \\
\text { NCT02528214 }\end{array}$ & $\begin{array}{l}\text { Glucocorticoid-dependent } \\
\text { severe asthma }\end{array}$ & $\mathrm{n} / \mathrm{a}$ & $\begin{array}{l}\text { High-dose ICS (FP } \\
\quad 500 \mu \mathrm{g} \text { per day or } \\
\text { equivalent)+up to } 2 \\
\text { additional } \\
\text { controllers } \\
\text { +maintenance OCS } \\
\text { lequivalent to } \\
\text { prednisone } 5- \\
35 \text { mg per dayl }\end{array}$ & $\begin{array}{l}\geqslant 12 \% \text { and } 200 \mathrm{~mL} \text {, or } \\
\text { airway hyper- } \\
\text { responsiveness }\end{array}$ & $\mathrm{n} / \mathrm{a}$ & n/a & n/a & $\begin{array}{l}\text { Current smokers, or } \\
\text { former smokers } \\
\text { with >10 pack-years; } \\
\text { COPD or other lung } \\
\text { disease that may } \\
\text { impair lung } \\
\text { function; clinically } \\
\text { significant lung } \\
\text { disease other than } \\
\text { asthma }\end{array}$ & $\begin{array}{l}\text { \% reduction in OCS } \\
\text { dose while } \\
\text { maintaining asthma } \\
\text { control }^{\# \#}\end{array}$ \\
\hline $\begin{array}{l}\text { STRATOS 1, } \\
\text { STRATOS } 2 \text { [110] } \\
\text { (tralokinumab) } \\
\text { NCT02161757, } \\
\text { NCT02194699 }\end{array}$ & $\begin{array}{l}\text { Severe, uncontrolled } \\
\text { asthma }\end{array}$ & $\begin{array}{l}\text { ACQ- } 6 \text { score } \geqslant 1.5 ; \\
\geqslant 2 \text { exacerbations } \\
\text { requiring OCS in } \\
\text { the past year [116] }\end{array}$ & $\begin{array}{l}\text { High-dose ICS (FP } \\
\geqslant 500 \mu \mathrm{g} \text { per day or } \\
\text { equivalent)+LABA } \\
\text { tadditional } \\
\text { controllers } \\
\text { excluding OCS }\end{array}$ & $\geqslant 12 \%$ and $\geqslant 200 \mathrm{~mL}$ & $\mathrm{n} / \mathrm{a}$ & $\begin{array}{l}\geqslant 37 \mathrm{ppb} \text { in STRATOS } 2 \\
\text { primary analysis } \\
\text { population }\end{array}$ & $\mathrm{n} / \mathrm{a}$ & $\begin{array}{l}\text { Regular OCS use within } \\
\text { past } 3 \text { months; } \\
\text { current smokers, or } \\
\text { former smokers } \\
\text { with } \geqslant 10 \\
\text { pack-years; } \\
\text { clinically important } \\
\text { pulmonary disease } \\
\text { other than asthma } \\
{[116]}\end{array}$ & $\begin{array}{l}\text { AER in all-comers } \\
\text { (STRATOS 1) or } \\
\text { patients with Feno } \\
\geqslant 37 \text { ppb } \\
\text { (STRATOS 2) }\end{array}$ \\
\hline
\end{tabular}




\begin{tabular}{|c|c|c|c|c|c|c|c|c|c|}
\hline Trial (treatment) & $\begin{array}{l}\text { Authors' description of } \\
\text { target population }\end{array}$ & Severity/control & Current treatment & $\begin{array}{l}\text { Bronchodilator } \\
\quad \text { reversibility } \\
\text { requirement (see } \\
\text { footnotes) }\end{array}$ & Eosinophilic status & Feno requirement & $\begin{array}{l}\text { Allergy/atopy } \\
\text { requirement }\end{array}$ & Notable exclusions & Primary endpoint \\
\hline $\begin{array}{l}\text { TROPOS [111] } \\
\text { (tralokinumab) } \\
\text { NCT02281357 }\end{array}$ & $\begin{array}{l}\text { Severe, uncontrolled } \\
\text { asthma }\end{array}$ & $\begin{array}{l}\text { Severe, uncontrolled } \\
\text { asthma requiring } \\
\text { maintenance OCS } \\
\text { treatment plus ICS/ } \\
\text { LABAs }\end{array}$ & $\begin{array}{l}\text { Medium- to high-dose } \\
\text { ICS (FP } \geqslant 500 \mu g \\
\text { per day or } \\
\text { equivalent]+LABA } \\
\text { + maintenance OCS } \\
\text { lequivalent to } \\
\text { prednisone } 7.5- \\
30 \mathrm{mg} \text { per day) }\end{array}$ & $\begin{array}{l}\geqslant 12 \% \text { or documented } \\
\text { reversibility in the } \\
\text { past } 6 \text { months }\end{array}$ & $\mathrm{n} / \mathrm{a}$ & $\mathrm{n} / \mathrm{a}$ & n/a & $\begin{array}{l}\text { Current smokers, or } \\
\text { former smokers } \\
\text { with } \geqslant 10 \\
\text { pack-years; } \\
\text { clinically important } \\
\text { pulmonary disease } \\
\text { other than asthma } \\
\text { (including COPD) }\end{array}$ & $\begin{array}{l}\% \text { reduction in OCS } \\
\text { dose while } \\
\text { maintaining asthma } \\
\text { control } \text { III }^{\text {If }}\end{array}$ \\
\hline \multicolumn{10}{|c|}{ Target population: patients with COPD } \\
\hline $\begin{array}{l}\text { METREX, METREO } \\
\text { [33] } \\
\text { (mepolizumab) } \\
\text { NCT02105948, } \\
\text { NCT02105961 }\end{array}$ & Eosinophilic COPD & $\begin{array}{l}\mathrm{FEV}_{1} / \mathrm{FVC}<0.7 \text { and } \\
\text { post-bronchodilator } \\
\mathrm{FEV}_{1}>20 \% \text { and } \\
\leqslant 80 \% \text { predicted; } \\
\geqslant 2 \text { moderate or } \\
\geqslant 1 \text { severe } \\
\text { exacerbations in } \\
\text { past year }\end{array}$ & $\begin{array}{l}\text { High-dose ICS (FP } \\
\geqslant 500 \mu \mathrm{\mu g} \text { per day or } \\
\text { equivalent)+LABA } \\
\text { +LAMA }\end{array}$ & n/a & $\begin{array}{l}\text { METREX: no blood } \\
\text { eosinophil } \\
\text { threshold } \\
\text { METREO: blood } \\
\text { eosinophil count of } \\
\geqslant 300 \text { cells per } \mu \mathrm{L} \\
\text { in previous } \\
12 \text { months or of } \\
\geqslant 150 \text { cells per } \mu \mathrm{L} \\
\text { at screening }\end{array}$ & n/a & n/a & $\begin{array}{l}\text { Current diagnosis of } \\
\text { asthma; any history } \\
\text { of asthma in never } \\
\text { smokers; age } \\
<40 \text { years }\end{array}$ & $\begin{array}{l}\text { Moderate/severe AER } \\
\text { In METREX, all } \\
\text { patients and } \\
\text { eosinophilic } \\
\text { ( } 300 \text { cells per } \mu \mathrm{L} \text { in } \\
\text { previous } 12 \text { months } \\
\text { or } \geqslant 150 \text { cells per } \mu \mathrm{L} \\
\text { at screening) } \\
\text { patients were } \\
\text { analysed as } \\
\text { separate groups }\end{array}$ \\
\hline $\begin{array}{l}\text { GALATHEA, } \\
\text { TERRANOVA [34] } \\
\text { (benralizumab) } \\
\text { NCT02155660, } \\
\text { NCT02138916 }\end{array}$ & $\begin{array}{l}\text { Moderate-to-very severe } \\
\text { COPD with } \\
\text { exacerbation history }\end{array}$ & $\begin{array}{l}\text { Post-bronchodilator } \\
\text { FEV }>20 \% \text { and } \\
\leqslant 65 \% \text { predicted; } \\
\geqslant 2 \text { exacerbations } \\
\text { requiring OCS or } \\
\text { antibiotics or } \geqslant 1 \\
\text { requiring } \\
\text { hospitalisation in } \\
\text { the past year; } \\
\text { mMRC score } \geqslant 1\end{array}$ & $\begin{array}{l}\text { LABA+LAMA and/or } \\
\text { ICS }\end{array}$ & n/a & $\begin{array}{l}\text { No blood eosinophil } \\
\text { threshold, but } \\
\text { enrolment } \\
\text { stratified/capped } \\
\text { by blood eosinophil } \\
\text { count }(\geqslant 220 \text { cells } \\
\text { per } \mu L \text { in primary } \\
\text { analysis } \\
\text { population) }\end{array}$ & $\mathrm{n} / \mathrm{a}$ & n/a & $\begin{array}{l}\text { Non-smokers or } \\
\text { smoking history < } 10 \\
\text { pack-years; } \\
\text { clinically important } \\
\text { pulmonary disease } \\
\text { other than COPD; } \\
\text { asthma as a primary } \\
\text { or main diagnosis; } \\
\text { age < } 40 \text { years }\end{array}$ & $\begin{array}{l}\text { AER in patients with } \\
\text { baseline blood } \\
\text { eosinophils } \\
\geqslant 220 \text { cells per } \mu \mathrm{L}\end{array}$ \\
\hline
\end{tabular}

Where publications state "systemic corticosteroid", it is assumed for the purposes of this review that they refer mostly or entirely to patients receiving OCS. RCT: randomised controlled trial; FeNO: exhaled nitric oxide fraction; ICS: inhaled corticosteroid; BDP: beclomethasone dipropionate; n/a: not applicable (not mentioned in inclusion/exclusion criteria); OCS: oral corticosteroid; PAR: persistent allergic rhinitis; AQLQ: Asthma Quality of Life Questionnaire; RQLQ: Rhinitis Quality of Life Questionnaire; ER: emergency room; GINA: Global Initiative for Asthma; PEF: peak expiratory flow; LABA: long-acting $\beta_{2}$-agonist; FP: fluticasone propionate; SABA: short-acting $\beta_{2}$-agonist; ERS: European Respiratory Society; ATS: American Thoracic Society; SGRQ: St George's Respiratory Questionnaire; COPD: chronic obstructive pulmonary disease; ACQ-n: $n$-item Asthma Control Questionnaire; AER: annual exacerbation rate; FEV forced expiratory volume in $1 \mathrm{~s}$; FVC: forced vital capacity; LAMA: long-acting muscarinic antagonist; mMRC: modified Medical Research Council dyspnoea scale. \#: published information does not state whether the dose range given for current treatment inclusion criterion was metered or delivered dose [91]; ${ }^{9}:$ one of $\geqslant 12 \%$ reversibility, positive results on methacholine or mannitol challenge, or $\mathrm{FEV}_{1}$ variability $(\geqslant 20 \%)$ between two visits $[63,101]$; ${ }^{+}$: one of $\geqslant 12 \%$ and $200 \mathrm{~mL}$ reversibility, positive results on methacholine or mannitol challenge, FEV, variability $(\geqslant 20 \%)$ between two visits, or $>20 \%$ diurnal variability in peak flow [67]; ${ }^{\S}$ : dose reduction was mandatory unless patients had an exacerbation, met any criteria for loss of asthma control (PEF, night-time awakenings, rescue medication use and ACQ-5 score) or had symptoms of adrenal insufficiency [62]; $f$ : dose reduction was mandatory unless patients had worsening of asthma symptoms (new or increased asthma symptoms or clinical signs that were troubling to the patient or were related to an electronic Asthma Daily Diary alert upon reduction) or did not meet reduction criteria (pre-bronchodilator FEV 1, PEF, night-time awakenings and rescue medication use); ${ }^{\# \#}$ : defined as the lowest dose that a patient could receive without having an increase in ACQ-5 score of $\geqslant 0.5$, a severe exacerbation or any clinically significant event leading to an upward adjustment in the oral glucocorticoid dose [64]; กा: defined as the lowest dose that a patient could receive while meeting all reduction criteria (pre-bronchodilator FEV ${ }_{1}$, PEF, night-time awakenings, rescue medication use, no exacerbations requiring OCS and investigator judgement of asthma control) [111]. 


\section{Comorbidities}

Most of the asthma trials excluded patients with lung disease other than asthma, including COPD; this was most consistent among the more recent trials [62-64, 99, 101-111]. Additionally, most studies excluded patients with features more characteristic of COPD [14], such as a history of smoking [62, 94, 98, $100-102,110,111]$ or lack of bronchodilator reversibility [62-64,91-94, 96, 99-101, 103-111]. Conversely, all of the COPD trials excluded patients with a current or primary diagnosis of asthma, and most excluded non-smokers or patients with $<10$ pack-years [34, 112-114].

\section{Primary endpoints}

Primary endpoints varied between trials. The majority of trials specified exacerbation reduction as a primary endpoint. Six trials evaluated lung function (one as a co-primary endpoint with exacerbation reduction) $[95,99,104,105,109,115]$, two evaluated quality of life (one as a co-primary endpoint with exacerbation reduction) [93, 102], four evaluated OCS sparing $[62-64,111]$ and one evaluated target-specific biomarker expression [98].

\section{Biomarkers for predicting response to biologic therapy}

In addition to their primary analyses, several of the phase III trials included pre-specified or post hoc sub-analyses that identified biomarkers that predicted treatment response $[33,64,82,100,102,105-111$, $117,118]$ (summarised in table 3 ). In a post hoc analysis of INNOVATE for omalizumab, higher baseline IgE predicted a greater reduction in clinically significant exacerbations than in patients with lower baseline IgE [117], but this was not confirmed in a separate analysis [119]. A pre-specified post hoc analysis of T2 biomarkers in EXTRA found that higher FeNO, blood eosinophil count and periostin all predicted a greater exacerbation rate reduction with omalizumab than their respective low-biomarker subgroups [82], although potential suppression of eosinophils by corticosteroids [76] suggests that eosinophil count should be assessed in light of OCS and ICS exposure. In patients with asthma taking high-dose ICS, blood eosinophil count predicted response to mepolizumab for several endpoints based on exploratory modelling in DREAM [100] and MUSCA [102] and a pooled post hoc analysis of DREAM and MENSA [118], and blood eosinophil count similarly predicted response to mepolizumab in patients with COPD in a meta-analysis of METREX and METREO [33]. Likewise, blood eosinophil count predicted responses to reslizumab [105] and benralizumab [106, 107] in patients with asthma, except for exacerbation rate in CALIMA, potentially due to a large "placebo" response that may have resulted from background ICS being supplied to patients [106]. However, pre-specified subgroup analyses of the GALATHEA and TERRANOVA trials showed no association between blood eosinophil count and response to benralizumab in patients with COPD [34]. In the LAVOLTA trials for lebrikizumab, both eosinophil-high patients and a biomarker-high group with eosinophilia and high periostin showed greater exacerbation reduction than the respective "low" groups, while stratifying by eosinophilia alone showed the greatest difference in exacerbation rate [108]. In LIBERTY ASTHMA VENTURE [64] and LIBERTY ASTHMA QUEST [109], dupilumab efficacy for exacerbation reduction, $\mathrm{FEV}_{1}$ improvement or OCS sparing was greatest in patients with higher baseline blood eosinophil counts and/or FeNO. Similarly, higher FeNO predicted significant exacerbation reduction with tralokinumab in STRATOS 1, although this was not replicated in STRATOS 2 [110] and there was no difference in OCS sparing based on FeNO levels in TROPOS [111]. Though not a complete review of biomarker studies in the biologic era, the findings described above suggest that several biomarkers specific to T2 inflammation mechanisms can predict response to biologic therapies that target components of the T2 pathway. Although the most appropriate cut-off points are yet to be determined, this supports the concept that establishing molecularly defined endotypes will enable better characterisation of patients with severe obstructive lung disease to inform treatment decisions.

\section{Limitations of the current approach to trial design}

Our review of phase III RCTs of biologic therapies demonstrates that these trials have narrow and sometimes conflicting eligibility criteria that exclude certain phenotypes of interest (summarised in box 1). For example, most required bronchodilator reversibility at screening, despite this being more difficult to demonstrate once patients are taking maintenance treatment [14]. Such a requirement is illogical, because it requires patients with long-standing, chronic disease to continue to satisfy criteria by which the disease is diagnosed at the time of initial presentation. Many severe asthma trials excluded patients with another pulmonary disease (such as COPD), even though patients with asthma-COPD overlap comprise $15 \%-30 \%$ of patients with chronic airways disease $[5,120]$. Asthma trials also excluded current smokers and patients with $\geqslant 10$ pack-years' smoking history, who represent approximately $26 \%-32 \%$ of the severe asthma population $[35,121]$, whereas most COPD trials (including all of the phase II trials identified) excluded patients with $<10$ pack-years [34, 112-114]. Some patients with COPD display T2-high and/or eosinophilic phenotypes $[122,123]$, and those with eosinophilic COPD have been shown to respond to mepolizumab for moderate-to-severe exacerbations [33], albeit to a lesser extent than patients with 
TABLE 3 Biomarkers that predicted treatment response in phase III RCTs of biologic therapies in severe obstructive lung disease

\section{Cut-off (greater response versus lesser/no response)}

\section{Phase III RCTs in patients with severe asthma}

IgE

$\geqslant 274 \mathrm{IU} \cdot \mathrm{mL}^{-1}, 148-273 \mathrm{IU} \cdot \mathrm{mL}^{-1}$ and $76-147 \mathrm{IU} \cdot \mathrm{mL}^{-1}$ versus $0-75 \mathrm{IU} \cdot \mathrm{mL}^{-1}$

\section{Blood eosinophil count}

$\geqslant 260$ cells per $\mu \mathrm{L}$ versus $<260$ cells per $\mu \mathrm{L}$

Continuous modelling (higher blood eosinophil count = greater response)

Continuous modelling (higher blood eosinophil count = greater response); identified a cut-off of $\geqslant 150$ cells per $\mu \mathrm{L}$ versus $<150$ cells per $\mu \mathrm{L}$

Continuous modelling (higher blood eosinophil count = greater response)

$\geqslant 400$ cells per $\mu \mathrm{L}$ versus $<400$ cells per $\mu \mathrm{L}$

$\geqslant 300$ cells per $\mu \mathrm{L}$ versus $<300$ cells per $\mu \mathrm{L}$

$\geqslant 300$ cells per $\mu \mathrm{L}$ versus $<300$ cells per $\mu \mathrm{L}$

$\geqslant 300$ cells per $\mu \mathrm{L}$ versus $<300$ cells per $\mu \mathrm{L}$

$\geqslant 300$ cells per $\mu L$ and $\geqslant 150-<300$ cells per $\mu L$ versus $<150$ cells per $\mu L$

$\geqslant 300$ cells per $\mu \mathrm{L}$ versus $<300$ cells per $\mu \mathrm{L}$

\section{FeNO}

$\geqslant 19.5 \mathrm{ppb}$ versus $<19.5 \mathrm{ppb}$

$\geqslant 50 \mathrm{ppb}$ and $\geqslant 25-<50 \mathrm{ppb}$ versus $<25 \mathrm{ppb}$

$\geqslant 50 \mathrm{ppb}$ and $\geqslant 25-50 \mathrm{ppb}$ versus $<25 \mathrm{ppb}$

$\geqslant 37 \mathrm{ppb}$ versus $<37 \mathrm{ppb}$ or all patients

\section{Periostin}

$\geqslant 50 \mathrm{ng} \cdot \mathrm{mL}^{-1}$ versus $<50 \mathrm{ng} \cdot \mathrm{mL}^{-1}$

Combined blood eosinophil count+periostin

$\geqslant 300$ cells per $\mu \mathrm{L}$ or $\geqslant 50 \mathrm{ng} \cdot \mathrm{mL}^{-1}$ versus $<300$ cells per $\mu \mathrm{L}$ or $<50 \mathrm{ng} \cdot \mathrm{mL}^{-1}$

\section{Phase III RCTs in patients with severe COPD}

\section{Blood eosinophil count}

$\geqslant 500$ cells per $\mu \mathrm{L}, \geqslant 300-<500$ cells per $\mu \mathrm{L}$ and $\geqslant 150-<300$ cells per $\mu \mathrm{L}$ versus $>150$ cells per $\mu \mathrm{L}$

\section{Outcomels]}

\section{Trial (treatment)}

Exacerbation rate Emergency visits $\mathrm{FEV}_{1}$

AQLQ score

Exacerbation rate

Exacerbation rate

Exacerbation rate

Trends also noted for:

FEV 1

SGRQ score

ACQ-5 score

Exacerbation rate

FEV

ACQ-5 score

$\mathrm{FEV}_{1}$

Exacerbation rate

$\mathrm{FEV}_{1}$

Exacerbation rate $\mathrm{FEV}_{1}$

$\mathrm{FEV}_{1}$

OCS dose

Exacerbation rate $\mathrm{FEV}_{1}$

Exacerbation rate

$\mathrm{FEV}_{1}$

OCS dose

Exacerbation rate $\mathrm{FEV}_{1}$

Exacerbation rate $\mathrm{FEV}_{1}$

$A Q L Q$ score

ACQ-6 score

Total asthma

symptom score

Exacerbation rate

Exacerbation rate

Exacerbation rate
INNOVATE [117] (omalizumab)

EXTRA [82] (omalizumab)

DREAM [100] (mepolizumab)

DREAM/MENSA [118]

(mepolizumab)

MUSCA [102] (mepolizumab)

Corren et al. [105] (reslizumab)

LAVOLTA I and LAVOLTA II [108] (lebrikizumab)

CALIMA [106] (benralizumab)

SIROCCO [107] (benralizumab)

LIBERTY ASTHMA QUEST [109] (dupilumab)

LIBERTY ASTHMA VENTURE

[64] (dupilumab)

EXTRA [82] (omalizumab)

LIBERTY ASTHMA QUEST [109] (dupilumab)

LIBERTY ASTHMA VENTURE

[64] (dupilumab)

STRATOS 1 [110] (tralokinumab)

EXTRA [82] (omalizumab)

LAVOLTA I and LAVOLTA II [108] (lebrikizumab)

\section{METREX/ METREO [33]}

(mepolizumab)

RCT: randomised controlled trial; $\mathrm{FEV}_{1}$ : forced expiratory volume in $1 \mathrm{~s} ; \mathrm{AQLQ}$ : Asthma Quality of Life Questionnaire; FeNo: exhaled nitric oxide fraction; COPD: chronic obstructive pulmonary disease; SGRQ: St George's Respiratory Questionnaire; ACQ- $n$ : $n$-item Asthma Control Questionnaire; OCS: oral corticosteroid. 
BOX 1 Eligibility criteria that may exclude populations of interest from phase III randomised controlled trials of biologic therapies in severe obstructive lung disease

\section{Bronchodilator reversibility}

- May exclude patients in whom reversible airflow limitation is no longer apparent due to treatment

- Inappropriately requires patients with chronic disease to continue to satisfy criteria for initial diagnosis

- May exclude patients with asthma-chronic obstructive pulmonary disease (COPD) overlap, including patients with asthma and non-reversible airflow limitation or COPD and reversible airflow limitation

Comorbidities (respiratory and/or non-respiratory)

- Excludes patients with asthma-COPD overlap

- Excludes patients with persistent airway infection or other lung diseases

- Precludes research to identify endotypes in patients with multi-morbidity

Smoking history

- Excludes smokers with asthma and patients with COPD who have limited/no smoking history

- May exclude patients with asthma-COPD overlap

- Excludes patients with COPD with a phenotype/endotype that is relevant to a specific mechanism of action le.g. eosinophilic phenotype)

Disease severity/control

- Precludes investigation of the potential benefits of earlier intervention or treatment of milder disease

- Excludes patients whose obstructive lung disease appears less severe, but who depend on high-dose inhaled corticosteroids or maintenance oral corticosteroids for adequate control

eosinophilic asthma [118]. This suggests that significant subsets of patients with severe obstructive lung disease, who could potentially benefit from biologic therapies, are excluded from trials that inform regulatory decisions and thus influence treatment options in clinical practice. A recent analysis of patients with severe asthma found that only $3.5 \%-17.5 \%$ would have been eligible for enrolment in 14 phase III trials of biologic therapies in severe asthma [35]. Furthermore, comorbidity is an important contributor to disease burden in both asthma [124] and COPD [125, 126], and excluding patients with comorbidities from RCTs limits the evidence available to support treatment approaches that target multi-morbidity via underlying mechanisms. Additionally, although patients with severe, uncontrolled disease are the focus of most RCTs to date, evidence of benralizumab efficacy for pre-bronchodilator $\mathrm{FEV}_{1}$ in a short-term study in patients with milder but persistent asthma [127] suggests that earlier intervention with biologic therapy may prevent the early structural damage that contributes to the development of severe disease in some patients $[127,128]$.

\section{Recommendations for future research and regulatory indications of biologic therapies \\ The importance of accurately defining severe obstructive lung disease}

Given that long-term treatment with OCS or high-dose ICS can have potentially costly long-term adverse effects [52, 53, 55-57, 59], treatment with alternative controllers and/or targeted biologics (despite high acquisition costs) may be the preferred approach in patients with asthma who fail to achieve control with lower doses [21]. This is reflected in more recent clinical definitions of severe asthma, which include patients dependent on medium- to high-dose ICS/LABA with or without OCS to maintain control (i.e. asthma is uncontrolled on a medium dose) (table 1). However, most RCTs of biologic therapies in severe obstructive lung disease enrol patients whose asthma is uncontrolled on medium- to high-dose ICS, with or without additional controllers (table 2). This, together with the high acquisition costs [21], has led some regulators and payers to restrict the approved indications of such medications to patients whose asthma is inadequately controlled despite high-dose ICS plus LABA or additional controllers [22, 25, 27], thereby missing the opportunity to reduce long-term high-dose ICS and maintenance OCS use in patients who have achieved control with such treatment.

\section{An endotype-based approach to future RCTs}

The use of highly specific eligibility criteria in existing RCTs of biologic therapies in severe obstructive lung disease may exclude patients with clinically relevant phenotypes (box 1), thereby limiting the generalisability of such trials to patients in clinical practice. In countries with fewer restrictions for prescribing biologic therapies for obstructive lung disease, real-world studies may reveal the extent to which RCT findings can be generalised to patients who do not fulfil typical inclusion criteria. To aid exploratory analyses and identify additional potentially responsive populations, we believe that trial populations (particularly for earlier phase studies) should include groups that are currently excluded, such 
as patients with persistent or latent airway infection or other lung diseases (e.g. bronchiectasis), patients with asthma and non-reversible airflow limitation, patients with cardiovascular and other comorbidities, and patients who have normal interval lung function but nonetheless experience symptoms and exacerbations. Also, trials should include assessments that may help to elucidate responsive phenotypes or endotypes, such as bronchoscopic evaluation. There is increasing interest in breathomics, which in a recent validation study identified clusters of patients with asthma/COPD that differed by ethnicity, systemic eosinophilia and neutrophilia, FeNO, body mass index, atopy and exacerbation rate, regardless of the diagnostic label [129]. In addition to identifying molecular biomarkers for targeted biologic therapies, such an approach could also be applied to RCTs of emerging non-pharmacological treatments, such as bronchoscopic lung reduction in patients with emphysema-predominant COPD [45] and bronchial thermoplasty in patients with severe asthma [14]. For example, although the mechanism of clinical benefit from bronchial thermoplasty is currently not well defined, it has been suggested that structural features measured by high resolution imaging, e.g. airway smooth muscle mass, could be used to characterise severe asthma phenotypes and predict response [130]. Future studies to identify biological predictors of response to such treatments could enable a wider array of treatment options to be included in the personalised healthcare repertoire for severe obstructive lung disease. Ultimately, for the maximum number of patients to gain access to the most appropriate treatment, a paradigm shift is likely to be required in patient selection for trials, moving away from conventional diagnostic labels and control criteria (clinical approach) towards recruitment and stratification of clinically broader populations predicted to respond based on an underlying, biologically defined disease mechanism (endotype-based approach).

This endotype-based approach is not yet recognised by regulators, and the consequent risk to pharmaceutical developers of failing to satisfy current approval requirements may deter them from conducting studies in this way. However, if there is sound scientific rationale underpinning the decision to target a specific population, based on endotype and drug mechanism of action rather than conventional labels (supported by robust early-phase clinical development), it seems reasonable to predict that the probability of achieving successful treatment outcomes in phase III RCTs would be high. An additional benefit of this exploratory approach is the potential to identify reliable, lower-cost surrogates for exacerbations as the primary outcome. In our opinion, pharmaceutical developers should be able to adopt this endotype-based approach when defining eligibility criteria for future RCTs, to support regulatory approval and to provide evidence for clinical practice guidelines. This requires recognition of the value of such an approach by regulators so that more exploratory studies can meet approval requirements. Therefore, co-ordinated partnerships between investigators, pharmaceutical developers and regulators are necessary to make meaningful change and provide more patients with targeted treatment options.

In addition to this shift towards endotype-based enrolment, standardisation of eligibility criteria and outcome measures will be important in evaluating the therapeutic benefit of new biologics in the appropriate populations. To ensure the clinical benefit of such biologics, the targeted molecular endotype should manifest as a clinically important outcome, such as exacerbations. Developing a core outcome set could help to improve comparability between trials and ensure clinical relevance of trial data [131].

\section{Identifying novel endotypes in severe obstructive lung disease}

Existing treatments for severe obstructive lung disease, especially corticosteroids, inhibit inflammation via multiple targets and may have unwanted additional anti-inflammatory effects. There is now extensive evidence that molecularly targeted biologic therapies improve outcomes in patients with T2-high, inflammatory asthma that is inadequately controlled by medium- to high-dose ICS [62, 63, 91-104, 106, $107,132]$. However, not all targets evaluated in phase III trials have proven effective. For example, results for therapies targeting IL-13 have been mixed. Lebrikizumab significantly reduced exacerbation rate among "biomarker-high" patients with uncontrolled asthma in LAVOLTA I, but efficacy did not reach significance in LAVOLTA II [108].

In contrast, tralokinumab failed to significantly reduce exacerbation rate either in all-comers with severe asthma in STRATOS 1 [110] and TROPOS [111] or among FeNO-high patients in STRATOS 2 [110]; by contrast, in a recent phase II trial it significantly reduced FeNO and IgE levels, but not eosinophil counts, suggesting a non-eosinophil-mediated mechanism of action [133]. The anti-IL-5 receptor therapy benralizumab has shown efficacy in severe eosinophilic asthma $[63,106,107]$, but did not significantly reduce exacerbations in patients with eosinophilic COPD [34]. The failure of these phase III trials suggests that further research is needed to link phenotypes with molecularly defined, targetable endotypes, particularly in severe COPD and asthma-COPD overlap, where few data are available.

Despite mixed results for some therapies, trial success in patients with severe, T2-high asthma demonstrates that targeting specific endotypes could improve outcomes in other, less well-studied 
populations, such as patients with T2-low disease. Currently, all approved biologic therapies for severe obstructive lung disease target severe or moderate-to-severe asthma with T2 inflammation (either IgE-mediated, eosinophilic or OCS-dependent asthma) [22-30]. However, these patients may have one or more of various T2-high phenotypes, which may or may not include blood and/or airway eosinophilia $[134,135]$. Furthermore, up to $50 \%$ of patients with severe asthma lack T2 inflammation $[121,136,137]$, i.e. they have a T2-low phenotype (or their T2 inflammation is controlled by anti-inflammatory medication(s) [138]). Additionally, patients with lung disease other than asthma (e.g. COPD or asthmaCOPD overlap) can also have uncontrolled disease despite high-level treatment [139-141]. This heterogeneity results in an unmet need for targeted therapies that address the underlying causes of disease for patients with T2-low severe asthma or other phenotypes of severe obstructive lung disease not currently catered for by available biologics. Although our literature review focused on phase III trials, several non-T2-targeted biologic therapies have been investigated in earlier phases of clinical development. For example, a phase II trial of the anti-IL-17 receptor therapy brodalumab, which used similar eligibility criteria to most of the asthma studies listed in table 2 but did not differentiate patients based on inflammatory phenotype, failed to meet its primary endpoint of clinically meaningful improvement in Asthma Control Questionnaire total score (although a pre-specified subgroup analysis found a significant improvement among patients with high reversibility) [142]. Earlier trials of the anti-tumour necrosis factor- $\alpha$ therapies golimumab and etanercept were similarly unsuccessful $[143,144]$, but imatinib, an inhibitor of the stem cell factor receptor KIT, has shown promise in an early, placebo-controlled, proof-of-principle trial [145]. One therapy currently in development for the treatment of uncontrolled asthma, tezepelumab, may also be effective in T2-low disease. Tezepelumab is a thymic stromal lymphopoietin-targeted therapy that demonstrated efficacy regardless of blood eosinophil count $(<250$ cells per $\mu \mathrm{L}$ versus $\geqslant 250$ cells per $\mu \mathrm{L})$ in a phase IIb severe asthma trial [146], leading to it being granted Breakthrough Therapy Designation by the US Food and Drug Administration [32]. Defining severe obstructive lung disease and designing future trials in a way that maximises the potential therapeutic impact of existing and future biologic therapies will be key to finding more therapies that fulfil this need. Furthermore, identifying novel endotypes of obstructive lung disease, including those not involving T2 inflammation, should be a key goal of future research.

The current high cost of biologic therapies (versus the relatively low cost of OCS/ICS) makes accurate prediction and monitoring of response necessary. Previous research shows that endotype-specific biomarkers of T2 inflammation can predict a patient's response to biologic therapies that target these particular mechanisms. Future biomarkers identified and utilised for this purpose should, therefore, be appropriate to the endotype being treated, as recommended by previous cost-effectiveness studies [65]; however, substantial price discounts may be needed to achieve acceptable cost-effectiveness, even within biomarker-targeted populations [65].

To better understand the mechanisms underlying obstructive lung disease and to identify specific endotypes that may be carried forward into interventional studies, large-scale studies in broad, real-world populations with standardised outcome measures are needed. Studies such as U-BIOPRED [47] in asthma and ECLIPSE [147], SPIROMICS [148] and COPDGene [149] in COPD have yielded important insights in their respective populations [150-154], with the caveat that these cohorts each focus primarily on a single diagnostic label (U-BIOPRED did not exclude patients with COPD, but required an asthma diagnosis and excluded patients with a primary diagnosis of severe emphysema or bronchiectasis [47]). NOVELTY (a NOVEL observational longiTudinal studY in patients with a diagnosis or suspected diagnosis of asthma and/or COPD) is an ongoing study that includes approximately 12000 patients across the spectrum of obstructive lung disease, with broad inclusion criteria and very few exclusion criteria to capture a broad patient population [155]. In NOVELTY, patients are required to have a diagnosis or clinically suspected diagnosis of asthma and/or COPD (according to the treating physician), be aged $\geqslant 12$ years and be able to provide informed consent. The only exclusion criteria are participation in an interventional respiratory clinical trial in the previous 12 months, low likelihood of completing 3 years of follow-up and a primary respiratory diagnosis other than asthma or COPD (though co-diagnoses of other respiratory diseases are allowed) [155]. NOVELTY is prospectively collecting data on a wide range of diagnosis-agnostic variables, with the aim of identifying phenotypes and endotypes through detailed clinical and biomarker characterisation [155]. Such large observational studies will complement the RCT evidence base and may help to identify novel endotypes that can inform the development and use of future targeted therapies.

\section{Conclusions}

Current treatment recommendations for severe obstructive lung disease, based on high-dose ICS with one or more add-on therapies, are inadequate in some patients and can have long-term adverse effects. OCS, previously the mainstay of severe asthma treatment and still used in frequent pulses for the treatment of 
severe exacerbations, has for some time been recognised as having serious, often permanent, adverse effects. Alternative, biologic therapies are currently only available for patients with T2-high phenotypes. Additionally, the narrow eligibility criteria used in existing RCTs of these therapies mean that their generalisability is limited to patients with specific clinical phenotypes, leading to limited therapeutic reach owing to regulatory restrictions. An unmet need, therefore, remains in two areas:

1) Studies of existing biologics in patients typically excluded from RCTs, including those whose asthma is well controlled on high-dose ICS and those with overlapping diagnostic labels (e.g. asthma and COPD), to provide evidence to support regulatory approval and reimbursement in such populations.

2) Targeted biologic therapies (and biomarkers to predict response) for patients with severe obstructive lung disease that is not, or is only partially, driven by T2 inflammation.

We therefore recommend a phenotype- and endotype-focused approach to future research on severe obstructive lung disease, in both clinical trials and exploratory studies, to identify novel biomarkers and potential targets. The success of this approach will depend on co-ordinated efforts between investigators, pharmaceutical developers and regulators to ensure biologic therapies reach their full potential in the treatment of patients with severe obstructive lung disease, irrespective of conventional diagnostic labels.

Acknowledgements: Medical writing support, under the direction of the authors, was provided by Nina Divorty, PhD, of CMC Connect, a division of McCann Health Medical Communications Ltd, Glasgow, UK, funded by AstraZeneca, Cambridge, UK, in accordance with Good Publication Practice (GPP3) guidelines (Ann Intern Med 2015; 163: 461464). All authors contributed to the conception of the article, interpretation of the literature review and development of the manuscript, and approved the final draft.

Conflict of interest: R.J. Martin reports grants from NHLBI, MedImmune and Chiesi Farmaceutici SpA, personal fees for steering committee work from AstraZeneca, personal fees for consultancy from PMD Healthcare, personal fees (honorarium) from Regeneron, and personal fees for advisory board work from Boehringer Ingelheim, outside the submitted work; he is a member of the NOVELTY Study Scientific Committee. E.H. Bel reports that the study and medical writing support was funded by AstraZeneca, during the conduct of the study; grants and personal fees from AstraZeneca, GSK, Novartis and Teva, and personal fees from Boehringer Ingelheim, Sanofi/Regeneron and Vectura, outside the submitted work; she is a member of the NOVELTY Study Scientific Committee. I.D. Pavord reports speaker's honoraria, travel expenses and honoraria for attending advisory boards from AstraZeneca, GSK, Boehringer Ingelheim and Teva, grants and speaker's honoraria, travel expenses and honoraria for attending advisory boards from Chiesi, personal fees for advisory board work from Sanofi/Regeneron, Merck, Novartis, Knopp and Roche/Genentech, personal fees for lectures from Circassia and Mundipharma, and grants and personal fees for advisory board work from Afferent, outside the submitted work; he is a member of the NOVELTY Study Scientific Committee. D. Price reports that the study was funded by AstraZeneca; grants and personal fees for advisory board membership and travel/ accommodation/meeting expenses from Aerocrine, grants from AKL Research and Development Ltd, British Lung Foundation, Respiratory Effectiveness Group and UK National Health Service, personal fees for consultancy and lectures from Almirall and GlaxoSmithKline, personal fees for advisory board membership and consultancy from Amgen, grants and personal fees for advisory board membership, consultancy, lectures and travel/accommodation/meeting expenses from AstraZeneca, Boehringer Ingelheim and Chiesi, personal fees for lectures from Cipla, Kyorin, Merck and Skyepharma, grants and personal fees for advisory board membership, consultancy and lectures from Mylan, grants and personal fees for advisory board membership, consultancy, lectures, manuscript preparation, educational activities and travel/accommodation/meeting expenses from Mundipharma, grants and personal fees for advisory board membership, consultancy and travel/accommodation/meeting expenses from Napp, grants and personal fees for advisory board membership, consultancy, lectures, patient enrolment or completion of research, development of educational materials and travel/accommodation/meeting expenses from Novartis, grants and personal fees for consultancy and lectures from Pfizer, grants and personal fees for advisory board membership and lectures from Regeneron Pharmaceuticals and Sanofi Genzyme, grants and personal fees for advisory board membership, consultancy, lectures, manuscript preparation, patient enrolment or completion of research and travel/accommodation/meeting expenses from Teva, grants and personal fees for consultancy from Theravance, and grants and personal fees for patient enrolment or completion of research from Zentiva (Sanofi Generics), and has participated in peer review for grant committees for Efficacy and Mechanism Evaluation programme and Health Technology Assessment, outside the submitted work; has stock/stock options from AKL Research and Development Ltd which produces phytopharmaceuticals; and owns $74 \%$ of the social enterprise Optimum Patient Care Ltd (Australia and UK) and 74\% of Observational and Pragmatic Research Institute Pte Ltd (Singapore); he is a member of the NOVELTY Study Scientific Committee. H.K. Reddel reports grants, personal fees and non-financial support from AstraZeneca and GlaxoSmithKline, and personal fees from Boehringer Ingelheim, Merck, Novartis, Teva and Mundipharma, outside the submitted work; she is a member of the NOVELTY Study Scientific Committee.

\section{References}

1 CIBA Guest Symposium. Terminology, definitions, and classification of chronic pulmonary emphysema and related conditions: a report of the conclusions of a CIBA guest symposium. Thorax 1959; 14: 286-299.

2 Fletcher CM, Pride NB. Definitions of emphysema, chronic bronchitis, asthma, and airflow obstruction: 25 years on from the CIBA symposium. Thorax 1984; 39: 81-85.

3 American Thoracic Society. Statement on standards for the diagnosis and care of patients with chronic obstructive pulmonary disease (COPD) and asthma. Am Rev Respir Dis 1987; 136: 225-244.

4 American Thoracic Society. Standards for the diagnosis and care of patients with chronic obstructive pulmonary disease. Am J Respir Crit Care Med 1995; 152: S77-121. 
Bateman ED, Reddel HK, van Zyl-Smit RN, et al. The asthma-COPD overlap syndrome: towards a revised taxonomy of chronic airways diseases? Lancet Respir Med 2015; 3: 719-728.

6 Reddel HK. Treatment of overlapping asthma-chronic obstructive pulmonary disease: can guidelines contribute in an evidence-free zone? J Allergy Clin Immunol 2015; 136: 546-552.

7 Agustí A, Bel E, Thomas M, et al. Treatable traits: toward precision medicine of chronic airway diseases. Eur Respir J 2016; 47: 410-419.

Moore WC, Fitzpatrick AM, Li X, et al. Clinical heterogeneity in the Severe Asthma Research Program. Ann Am Thorac Soc 2013; 10: Suppl., S118-S124.

9 Moore WC, Meyers DA, Wenzel SE, et al. Identification of asthma phenotypes using cluster analysis in the Severe Asthma Research Program. Am J Respir Crit Care Med 2010; 181: 315-323.

10 Chung KF, Wenzel SE, Brozek JL, et al. International ERS/ATS guidelines on definition, evaluation and treatment of severe asthma. Eur Respir J 2014; 43: 343-373.

11 Quaderi SA, Hurst JR. The unmet global burden of COPD. Glob Health Epidemiol Genom 2018; 3: e4.

12 Global Asthma Network. The Global Asthma Report 2018. http://globalasthmareport.org/Global\%20Asthma\% 20Report\%202018.pdf. Date last accessed: September 21, 2018.

13 Price D, Fletcher M, van der Molen T. Asthma control and management in 8,000 European patients: the REcognise Asthma and LInk to Symptoms and Experience (REALISE) survey. NPJ Prim Care Respir Med 2014; 24 : 14009.

14 Global Initiative for Asthma. Global Strategy for Asthma Management and Prevention (updated 2019). https:/ ginasthma.org/gina-reports/. Date last accessed: July 2, 2019.

15 Hekking PP, Wener RR, Amelink M, et al. The prevalence of severe refractory asthma. J Allergy Clin Immunol 2015; 135: 896-902.

16 Price D, West D, Brusselle G, et al. Management of COPD in the UK primary-care setting: an analysis of real-life prescribing patterns. Int J Chron Obstruct Pulmon Dis 2014; 9: 889-904.

17 Vestbo J, Papi A, Corradi M, et al. Single inhaler extrafine triple therapy versus long-acting muscarinic antagonist therapy for chronic obstructive pulmonary disease (TRINITY): a double-blind, parallel group, randomised controlled trial. Lancet 2017; 389: 1919-1929.

18 Sadatsafavi M, Lynd L, Marra C, et al. Direct health care costs associated with asthma in British Columbia. Can Respir J 2010; 17: 74-80.

19 Punekar YS, Shukla A, Müllerova H. COPD management costs according to the frequency of COPD exacerbations in UK primary care. Int J Chron Obstruct Pulmon Dis 2014; 9: 65-73.

20 Chen S, Golam S, Myers J, et al. Systematic literature review of the clinical, humanistic, and economic burden associated with asthma uncontrolled by GINA steps 4 or 5 treatment. Curr Med Res Opin 2018; 34: $2075-2088$.

Pavord ID, Beasley R, Agustí A, et al. After asthma: redefining airways diseases. Lancet 2018; 391: 350-400.

22 AstraZeneca UK Ltd. Fasenra (benralizumab) Summary of Product Characteristics. www.medicines.org.uk/emc/ product/8918/smpc. Date last accessed: June 6, 2018.

23 AstraZeneca Pharmaceuticals LP. Fasenra (benralizumab) Prescribing Information. www.azpicentral.com/fasenra/ fasenra_pi.pdf. Date last accessed: June 6, 2018.

24 Genentech USA Inc., Novartis Pharmaceuticals Corporation. Xolair (omalizumab) Prescribing Information. www.gene.com/download/pdf/xolair_prescribing.pdf. Date last accessed: October 16, 2018.

25 Novartis Pharmaceuticals UK Ltd. Xolair (omalizumab) Summary of Product Characteristics. www.medicines. org.uk/emc/product/4725/smpc. Date last accessed: June 6, 2018.

26 Teva Pharmaceutical Industries Ltd. Cinqair (reslizumab) Prescribing Information. www.cinqair.com/ globalassets/cinqair/prescribinginformation.pdf. Date last accessed: June 6, 2018.

27 Teva Pharmaceuticals Ltd. Cinqaero (reslizumab) Summary of Product Characteristics. www.medicines.org.uk/ emc/product/4370. Date last accessed: October 16, 2018.

28 GlaxoSmithKline. Nucala (mepolizumab) Prescribing Information. www.gsksource.com/pharma/content/dam/ GlaxoSmithKline/US/en/Prescribing_Information/Nucala/pdf/NUCALA-PI-PIL.PDF. Date last accessed: June 6, 2018.

29 GlaxoSmithKline UK. Nucala (mepolizumab) Summary of Product Characteristics. www.medicines.org.uk/emc/ product/1938. Date last accessed: October 16, 2018.

30 Sanofi-Aventis U.S. LLC, Regeneron Pharmaceuticals Inc. Dupixent (dupilumab) Prescribing Information. www. regeneron.com/sites/default/files/Dupixent_FPI.pdf. Date last accessed: October 25, 2018.

31 Wechsler ME. Current and emerging biologic therapies for asthma and COPD. Respir Care 2018; 63: 699-707.

32 AstraZeneca. Tezepelumab granted breakthrough therapy designation by US FDA. www.astrazeneca.com/ media-centre/press-releases/2018/tezepelumab-granted-breakthrough-therapy-designation-by-us-fda-07092018. html. Date last accessed: September 18, 2018.

33 Pavord ID, Chanez P, Criner GJ, et al. Mepolizumab for eosinophilic chronic obstructive pulmonary disease. $N$ Engl J Med 2017; 377: 1613-1629.

34 Criner GJ, Celli BR, Brightling CE, et al. Benralizumab for the prevention of COPD exacerbations. $N$ Engl J Med 2019; 381: 1023-1034.

35 Brown T, Jones T, Gove $\mathrm{K}$, et al. Randomised controlled trials in severe asthma: selection by phenotype or stereotype. Eur Respir J 2018; 52: 1801444.

36 Herland K, Akselsen JP, Skjonsberg OH, et al. How representative are clinical study patients with asthma or COPD for a larger "real life" population of patients with obstructive lung disease? Respir Med 2005; 99: 11-19.

37 Travers J, Marsh S, Caldwell B, et al. External validity of randomized controlled trials in COPD. Respir Med 2007; 101: 1313-1320.

38 Travers J, Marsh S, Williams M, et al. External validity of randomised controlled trials in asthma: to whom do the results of the trials apply? Thorax 2007; 62: 219-223.

39 Walker S, Fingleton J, Weatherall M, et al. Limited generalisability of UPLIFT findings to clinical practice. Thorax 2013; 68: 1066-1067.

40 Kruis AL, Ställberg B, Jones RCM, et al. Primary care COPD patients compared with large pharmaceutically-sponsored COPD studies: an UNLOCK validation study. PLoS One 2014; 9: e90145.

41 Scichilone N, Basile M, Battaglia S, et al. What proportion of chronic obstructive pulmonary disease outpatients is eligible for inclusion in randomized clinical trials? Respiration 2014; 87: 11-17. 
Halpin DMG, Kerkhof M, Soriano JB, et al. Eligibility of real-life patients with COPD for inclusion in trials of inhaled long-acting bronchodilator therapy. Respir Res 2016; 17: 120.

British Thoracic Society, Scottish Intercollegiate Guidelines Network. British Guideline on the Management of Asthma. www.brit-thoracic.org.uk/quality-improvement/guidelines/asthma/. Date last accessed: March 27, 2019. National Asthma Council Australia. Australian Asthma Handbook. www.asthmahandbook.org.au/. Date last accessed: May 24, 2018.

Global Initiative for Chronic Obstructive Lung Disease. Global Strategy for the Diagnosis, Management, and Prevention of Chronic Obstructive Pulmonary Disease (2019 Report). www.goldcopd.org. Date last accessed: March 21, 2019.

Lung Foundation Australia. Stepwise Management of Stable COPD. lungfoundation.com.au/wp-content/uploads/ 2018/09/Information-paper-Stepwise-Management-of-Stable-COPD-Aug2017.pdf. Date last accessed: May 24, 2018.

Shaw DE, Sousa AR, Fowler SJ, et al. Clinical and inflammatory characteristics of the European U-BIOPRED adult severe asthma cohort. Eur Respir J 2015; 46: 1308-1321.

Price DB, Trudo F, Voorham J, et al. Adverse outcomes from initiation of systemic corticosteroids for asthma: long-term observational study. J Asthma Allergy 2018; 11: 193-204.

Price D, Yawn B, Brusselle G, et al. Risk-to-benefit ratio of inhaled corticosteroids in patients with COPD. Prim Care Respir J 2013; 22: 92-100.

Bachert C, Zhang L, Gevaert P. Current and future treatment options for adult chronic rhinosinusitis: focus on nasal polyposis. J Allergy Clin Immunol 2015; 136: 1431-1440.

Ring J, Alomar A, Bieber T, et al. Guidelines for treatment of atopic eczema (atopic dermatitis) part I. J Eur Acad Dermatol Venereol 2012; 26: 1045-1060.

Lefebvre P, Duh MS, Lafeuille MH, et al. Acute and chronic systemic corticosteroid-related complications in patients with severe asthma. I Allergy Clin Immunol 2015; 136: 1488-1495.

Sarnes E, Crofford L, Watson M, et al. Incidence and US costs of corticosteroid-associated adverse events: a systematic literature review. Clin Ther 2011; 33: 1413-1432.

Sweeney J, Patterson CC, Menzies-Gow A, et al. Comorbidity in severe asthma requiring systemic corticosteroid therapy: cross-sectional data from the Optimum Patient Care Research Database and the British Thoracic Difficult Asthma Registry. Thorax 2016; 71: 339-346.

Voorham J, Xu X, Price D, et al. Health care resource utilization and costs associated with incremental systemic corticosteroid exposure in asthma. Allergy 2018; 67: 1-11.

Lipworth BJ. Systemic adverse effects of inhaled corticosteroid therapy: a systematic review and meta-analysis. Arch Intern Med 1999; 159: 941-955.

Suissa S, Kezouh A, Ernst P. Inhaled corticosteroids and the risks of diabetes onset and progression. Am J Med 2010; 123: 1001-1006.

Price DB, Russell R, Mares R, et al. Metabolic effects associated with ICS in patients with COPD and comorbid type 2 diabetes: a historical matched cohort study. PLoS One 2016; 11: e0162903.

Kew KM, Seniukovich A. Inhaled steroids and risk of pneumonia for chronic obstructive pulmonary disease. Cochrane Database Syst Rev 2014; 3: CD010115.

Sonnappa S, Martin R, Israel E, et al. Risk of pneumonia in obstructive lung disease: a real-life study comparing extra-fine and fine-particle inhaled corticosteroids. PLoS One 2017; 12: e0178112.

Roland NJ, Bhalla RK, Earis J. The local side effects of inhaled corticosteroids: current understanding and review of the literature. Chest 2004; 126: 213-219.

Bel EH, Wenzel SE, Thompson PJ, et al. Oral glucocorticoid-sparing effect of mepolizumab in eosinophilic asthma. N Engl J Med 2014; 371: 1189-1197.

Nair P, Wenzel S, Rabe KF, et al. Oral glucocorticoid-sparing effect of benralizumab in severe asthma. $N$ Engl $J$ Med 2017; 376: 2448-2458.

Rabe KF, Nair P, Brusselle G, et al. Efficacy and safety of dupilumab in glucocorticoid-dependent severe asthma. N Engl J Med 2018; 378: 2475-2485.

Queen RB, Sheehan DN, Whittington MD, et al. Cost-effectiveness of biological asthma treatments: a systematic review and recommendations for future economic evaluations. Pharmacoeconomics 2018; 36: 957-971. Bousquet J, Mantzouranis E, Cruz AA, et al. Uniform definition of asthma severity, control, and exacerbations: document presented for the World Health Organization Consultation on Severe Asthma. J Allergy Clin Immunol 2010; 126: 926-938.

Bel EH, Sousa A, Fleming L, et al. Diagnosis and definition of severe refractory asthma: an international consensus statement from the Innovative Medicine Initiative (IMI). Thorax 2011; 66: 910-917. it? Thorax 2009; 64: 728-735.

Cosio BG, Soriano JB, Lopez-Campos JL, et al. Defining the asthma-COPD overlap syndrome in a COPD cohort. Chest 2016; 149: 45-52.

Lopez-Campos JL, Peces-Barba G, Soler-Cataluna JJ, et al. Chronic obstructive pulmonary disease history assessment in Spain: a multidimensional chronic obstructive pulmonary disease evaluation. Study methods and organization. Arch Bronconeumol 2012; 48: 453-459.

Sin DD, Miravitlles M, Mannino DM, et al. What is asthma-COPD overlap syndrome? Towards a consensus definition from a round table discussion. Eur Respir J 2016; 48: 664-673.

Plaza V, Alvarez F, Calle M, et al. Consensus on the asthma-COPD overlap syndrome (ACOS) between the Spanish COPD Guidelines (GesEPOC) and the Spanish Guidelines on the Management of Asthma (GEMA). Arch Bronconeumol 2017; 53: 443-449.

Adams NP, Bestall JC, Jones P, et al. Fluticasone at different doses for chronic asthma in adults and children. Cochrane Database Syst Rev 2008; 4: CD003534.

moderate-to-severe asthma. Respir Med 2003; 97: 555-562.

75 Green RH, Brightling CE, McKenna S, et al. Asthma exacerbations and sputum eosinophil counts: a randomised controlled trial. Lancet 2002; 360: 1715-1721. 
Jayaram L, Pizzichini MM, Cook RJ, et al. Determining asthma treatment by monitoring sputum cell counts: effect on exacerbations. Eur Respir J 2006; 27: 483-494.

Kerkhof M, Tran TN, van den Berge M, et al. Association between blood eosinophil count and risk of readmission for patients with asthma: historical cohort study. PLoS One 2018; 13: e0201143.

Price D, Wilson AM, Chisholm A, et al. Predicting frequent asthma exacerbations using blood eosinophil count and other patient data routinely available in clinical practice. J Asthma Allergy 2016; 9: 1-12.

Ryan D, Blakey J, Chisholm A, et al. Use of electronic medical records and biomarkers to manage risk and resource efficiencies. Eur Clin Respir J 2017; 4: 1293386.

Bleecker ER, Wechsler ME, FitzGerald JM, et al. Baseline patient factors impact on the clinical efficacy of benralizumab for severe asthma. Eur Respir J 2018; 52: 1800936.

Price DB, Buhl R, Chan A, et al. Fractional exhaled nitric oxide as a predictor of response to inhaled corticosteroids in patients with non-specific respiratory symptoms and insignificant bronchodilator reversibility: a randomised controlled trial. Lancet Respir Med 2018; 6: 29-39.

Hanania NA, Wenzel S, Rosen K, et al. Exploring the effects of omalizumab in allergic asthma: an analysis of biomarkers in the EXTRA study. Am J Respir Crit Care Med 2013; 187: 804-811.

Kerkhof M, Freeman D, Jones R, et al. Predicting frequent COPD exacerbations using primary care data. Int $J$ Chron Obstruct Pulmon Dis 2015; 10: 2439-2450.

Martinez-Garcia MA, Miravitlles M. Bronchiectasis in COPD patients: more than a comorbidity? Int J Chron Obstruct Pulmon Dis 2017; 12: 1401-1411.

da Silva SM, Paschoal IA, De Capitani EM, et al. COPD phenotypes on computed tomography and its correlation with selected lung function variables in severe patients. Int J Chron Obstruct Pulmon Dis 2016; 11: 503-513.

Suissa S, Ernst P. Observational studies of inhaled corticosteroid effectiveness in COPD: lessons learned. Chest 2018; 154: 257-265.

Gershon AS, Campitelli MA, Croxford R, et al. Combination long-acting $\beta$-agonists and inhaled corticosteroids compared with long-acting $\beta$-agonists alone in older adults with chronic obstructive pulmonary disease. JAMA 2014; 312: 1114-1121.

Konno S, Taniguchi N, Makita H, et al. Distinct phenotypes of smokers with fixed airflow limitation identified by cluster analysis of severe asthma. Ann Am Thorac Soc 2018; 15: 33-41.

Konstantellou E, Papaioannou AI, Loukides S, et al. Persistent airflow obstruction in patients with asthma: characteristics of a distinct clinical phenotype. Respir Med 2015; 109: 1404-1409.

Muller V, Galffy G, Orosz M, et al. Characteristics of reversible and nonreversible COPD and asthma and COPD overlap syndrome patients: an analysis of salbutamol Easyhaler data. Int J Chron Obstruct Pulmon Dis 2016; 11: 93-101.

Busse W, Corren J, Lanier BQ, et al. Omalizumab, anti-IgE recombinant humanized monoclonal antibody, for the treatment of severe allergic asthma. J Allergy Clin Immunol 2001; 108: 184-190.

Solèr M, Matz J, Townley R, et al. The anti-IgE antibody omalizumab reduces exacerbations and steroid requirement in allergic asthmatics. Eur Respir J 2001; 18: 254-261.

Vignola AM, Humbert M, Bousquet J, et al. Efficacy and tolerability of anti-immunoglobulin E therapy with omalizumab in patients with concomitant allergic asthma and persistent allergic rhinitis: SOLAR. Allergy 2004; 59: 709-717.

Humbert M, Beasley R, Ayres J, et al. Benefits of omalizumab as add-on therapy in patients with severe persistent asthma who are inadequately controlled despite best available therapy (GINA 2002 step 4 treatment): INNOVATE. Allergy 2005; 60: 309-316.

Ohta K, Miyamoto T, Amagasaki T, et al. Efficacy and safety of omalizumab in an Asian population with moderate-to-severe persistent asthma. Respirology 2009; 14: 1156-1165.

Lanier B, Bridges T, Kulus M, et al. Omalizumab for the treatment of exacerbations in children with inadequately controlled allergic (IgE-mediated) asthma. J Allergy Clin Immunol 2009; 124: 1210-1216.

Hanania NA, Alpan O, Hamilos DL, et al. Omalizumab in severe allergic asthma inadequately controlled with standard therapy: a randomized trial. Ann Intern Med 2011; 154: 573-582.

Garcia G, Magnan A, Chiron R, et al. A proof-of-concept, randomized, controlled trial of omalizumab in patients with severe, difficult-to-control, nonatopic asthma. Chest 2013; 144: 411-419.

Li J, Kang J, Wang C, et al. Omalizumab improves quality of life and asthma control in Chinese patients with moderate to severe asthma: a randomized Phase III study. Allergy Asthma Immunol Res 2016; 8: 319-328.

Pavord ID, Korn S, Howarth P, et al. Mepolizumab for severe eosinophilic asthma (DREAM): a multicentre, double-blind, placebo-controlled trial. Lancet 2012; 380: 651-659.

Engl J Med 2014; 371: 1198-1207.

Chupp GL, Bradford ES, Albers FC, et al. Efficacy of mepolizumab add-on therapy on health-related quality of life and markers of asthma control in severe eosinophilic asthma (MUSCA): a randomised, double-blind, placebo-controlled, parallel-group, multicentre, phase 3b trial. Lancet Respir Med 2017; 5: 390-400.

3 Castro M, Zangrilli J, Wechsler ME, et al. Reslizumab for inadequately controlled asthma with elevated blood eosinophil counts: results from two multicentre, parallel, double-blind, randomised, placebo-controlled, phase 3 trials. Lancet Respir Med 2015; 3: 355-366.

Bjermer L, Lemiere C, Maspero J, et al. Reslizumab for inadequately controlled asthma with elevated blood eosinophil levels: a randomized phase 3 study. Chest 2016; 150: 789-798.

Corren J, Weinstein S, Janka L, et al. Phase 3 study of reslizumab in patients with poorly controlled asthma: effects across a broad range of eosinophil counts. Chest 2016; 150: 799-810.

FitzGerald JM, Bleecker ER, Nair P, et al. Benralizumab, an anti-interleukin-5 receptor alpha monoclonal antibody, as add-on treatment for patients with severe, uncontrolled, eosinophilic asthma (CALIMA): a randomised, double-blind, placebo-controlled phase 3 trial. Lancet 2016; 388: 2128-2141.

Bleecker ER, FitzGerald JM, Chanez P, et al. Efficacy and safety of benralizumab for patients with severe asthma uncontrolled with high-dosage inhaled corticosteroids and long-acting beta2-agonists (SIROCCO): a randomised, multicentre, placebo-controlled phase 3 trial. Lancet 2016; 388: 2115-2127. 
Hanania NA, Korenblat P, Chapman KR, et al. Efficacy and safety of lebrikizumab in patients with uncontrolled asthma (LAVOLTA I and LAVOLTA II): replicate, phase 3, randomised, double-blind, placebo-controlled trials. Lancet Respir Med 2016; 4: 781-796.

109 Castro M, Corren J, Pavord ID, et al. Dupilumab efficacy and safety in moderate-to-severe uncontrolled asthma. N Engl J Med 2018; 378: 2486-2496.

110 Panettieri RA Jr, Sjobring U, Peterffy A, et al. Tralokinumab for severe, uncontrolled asthma (STRATOS 1 and STRATOS 2): two randomised, double-blind, placebo-controlled, phase 3 clinical trials. Lancet Respir Med 2018; 6: 511-525.

111 Busse WW, Brusselle GG, Korn S, et al. Tralokinumab did not demonstrate oral corticosteroid-sparing effects in severe asthma. Eur Respir J 2019; 53: 1800948.

112 Rennard SI, Fogarty C, Kelsen S, et al. The safety and efficacy of infliximab in moderate to severe chronic obstructive pulmonary disease. Am J Respir Crit Care Med 2007; 175: 926-934.

113 Brightling CE, Bleecker ER, Panettieri RA Jr, et al. Benralizumab for chronic obstructive pulmonary disease and sputum eosinophilia: a randomised, double-blind, placebo-controlled, phase 2a study. Lancet Respir Med 2014; 2: 891-901.

114 Calverley PMA, Sethi S, Dawson M, et al. A randomised, placebo-controlled trial of anti-interleukin-1 receptor 1 monoclonal antibody MEDI8968 in chronic obstructive pulmonary disease. Respir Res 2017; 18: 153.

115 Eich A, Urban V, Jutel M, et al. A randomized, placebo-controlled phase 2 trial of CNTO 6785 in chronic obstructive pulmonary disease. COPD 2017; 14: 476-483.

116 Panettieri RA Jr, Brightling C, Sjobring U, et al. STRATOS 1 and 2: considerations in clinical trial design for a fully human monoclonal antibody in severe asthma. Clin Invest (Lond) 2015; 5: 701-711.

117 Bousquet J, Rabe K, Humbert $\mathrm{M}$, et al. Predicting and evaluating response to omalizumab in patients with severe allergic asthma. Respir Med 2007; 101: 1483-1492.

118 Ortega HG, Yancey SW, Mayer B, et al. Severe eosinophilic asthma treated with mepolizumab stratified by baseline eosinophil thresholds: a secondary analysis of the DREAM and MENSA studies. Lancet Respir Med 2016; 4: 549-556

119 Wahn U, Martin C, Freeman P, et al. Relationship between pretreatment specific IgE and the response to omalizumab therapy. Allergy 2009; 64: 1780-1787.

120 Alshabanat A, Zafari Z, Albanyan O, et al. Asthma and COPD overlap syndrome (ACOS): a systematic review and meta analysis. PLoS One 2015; 10: e0136065.

121 Schleich F, Brusselle G, Louis R, et al. Heterogeneity of phenotypes in severe asthmatics. The Belgian Severe Asthma Registry (BSAR). Respir Med 2014; 108: 1723-1732.

122 Christenson SA, Steiling K, van den Berge M, et al. Asthma-COPD overlap. Clinical relevance of genomic signatures of type 2 inflammation in chronic obstructive pulmonary disease. Am J Respir Crit Care Med 2015; 191: 758-766.

123 Singh D, Kolsum U, Brightling CE, et al. Eosinophilic inflammation in COPD: prevalence and clinical characteristics. Eur Respir J 2014; 44: 1697-1700.

124 Weatherburn CJ, Guthrie B, Mercer SW, et al. Comorbidities in adults with asthma: population-based cross-sectional analysis of 1.4 million adults in Scotland. Clin Exp Allergy 2017; 47: 1246-1252.

125 Lisspers K, Larsson K, Johansson G, et al. Economic burden of COPD in a Swedish cohort: the ARCTIC study. Int J Chron Obstruct Pulmon Dis 2018; 13: 275-285.

126 Vanfleteren LEGW, Spruit MA, Wouters EFM, et al. Management of chronic obstructive pulmonary disease beyond the lungs. Lancet Respir Med 2016; 4: 911-924.

127 Ferguson GT, FitzGerald JM, Bleecker ER, et al. Benralizumab for patients with mild to moderate, persistent asthma (BISE): a randomised, double-blind, placebo-controlled, phase 3 trial. Lancet Respir Med 2017; 5: $568-576$.

128 Coumou H, Westerhof GA, de Nijs SB, et al. Predictors of accelerated decline in lung function in adult-onset asthma. Eur Respir J 2018; 51: 1701785.

129 de Vries R, Dagelet YWF, Spoor P, et al. Clinical and inflammatory phenotyping by breathomics in chronic airway diseases irrespective of the diagnostic label. Eur Respir J 2018; 51: 1701817.

130 d'Hooghe JNS, ten Hacken NHT, Weersink EJM, et al. Emerging understanding of the mechanism of action of bronchial thermoplasty in asthma. Pharmacol Ther 2018; 181: 101-107.

131 Kirkham JJ, Davis K, Altman DG, et al. Core Outcome Set-STAndards for Development: The COS-STAD recommendations. PLoS Med 2017; 14: e1002447.

132 Corren J, Lemanske RF, Hanania NA, et al. Lebrikizumab treatment in adults with asthma. N Engl J Med 2011; 365: 1088-1098.

133 Russell RJ, Chachi L, FitzGerald JM, et al. Effect of tralokinumab, an interleukin-13 neutralising monoclonal antibody, on eosinophilic airway inflammation in uncontrolled moderate-to-severe asthma (MESOS): a multicentre, double-blind, randomised, placebo-controlled phase 2 trial. Lancet Respir Med 2018; 6: 499-510.

134 Good JT Jr, Kolakowski CA, Groshong SD, et al. Refractory asthma: importance of bronchoscopy to identify phenotypes and direct therapy. Chest 2012; 141: 599-606

135 Wenzel SE. Emergence of biomolecular pathways to define novel asthma phenotypes. type-2 immunity and beyond. Am J Respir Cell Mol Biol 2016; 55: 1-4.

136 Miranda C, Busacker A, Balzar S, et al. Distinguishing severe asthma phenotypes: role of age at onset and eosinophilic inflammation. J Allergy Clin Immunol 2004; 113: 101-108.

137 Wenzel SE. Severe asthma in adults. Exp Lung Res 2005; 31: Suppl. 1, 22.

138 Woodruff PG, Modrek B, Choy DF, et al. T-helper type 2-driven inflammation defines major subphenotypes of asthma. Am J Respir Crit Care Med 2009; 180: 388-395.

139 Mullerova $\mathrm{H}$, Maskell J, Meeraus WH, et al. Characterization of COPD patients treated with inhaled triple therapy containing inhaled corticosteroids [ICS], long-acting beta2-agonists [LABA], and long-acting muscarinic antagonists [LAMA] in the UK. Am J Respir Crit Care Med 2017; 195: A4986.

140 Yancey S, Albers FC, Gunsoy N, et al. Effect of mepolizumab on exacerbations in asthma patients with features common in COPD. Am J Respir Crit Care Med 2017; 195: A4683. 
141 Chen S, Small M, Lindner L, et al. Symptomatic burden of COPD for patients receiving dual or triple therapy. Int J Chron Obstruct Pulmon Dis 2018; 13: 1365-1376.

142 Busse WW, Holgate S, Kerwin E, et al. Randomized, double-blind, placebo-controlled study of brodalumab, a human anti-IL-17 receptor monoclonal antibody, in moderate to severe asthma. Am J Respir Crit Care Med 2013; 188: $1294-1302$.

143 Wenzel SE, Barnes PJ, Bleecker ER, et al. A randomized, double-blind, placebo-controlled study of tumor necrosis factor-alpha blockade in severe persistent asthma. Am J Respir Crit Care Med 2009; 179: 549-558.

144 Holgate ST, Noonan M, Chanez P, et al. Efficacy and safety of etanercept in moderate-to-severe asthma: a randomised, controlled trial. Eur Respir J 2011; 37: 1352-1359.

145 Cahill KN, Katz HR, Cui J, et al. KIT inhibition by imatinib in patients with severe refractory asthma. $N$ Engl J Med 2017; 376: 1911-1920.

146 Corren J, Parnes JR, Wang L, et al. Tezepelumab in adults with uncontrolled asthma. N Engl J Med 2017; 377: 936-946.

147 Vestbo J, Anderson W, Coxson HO, et al. Evaluation of COPD Longitudinally to Identify Predictive Surrogate End-points (ECLIPSE). Eur Respir J 2008; 31: 869-873.

148 Couper D, LaVange LM, Han M, et al. Design of the Subpopulations and Intermediate Outcomes in COPD Study (SPIROMICS). Thorax 2014; 69: 491-494

149 Regan EA, Hokanson JE, Murphy JR, et al. Genetic epidemiology of COPD (COPDGene) study design. COPD 2010; 7: 32-43.

150 Loza MJ, Adcock I, Auffray C, et al. Longitudinally stable, clinically defined clusters of patients with asthma independently identified in the ADEPT and U-BIOPRED asthma studies. Ann Am Thorac Soc 2016; 13: Suppl. 1, S102-S103.

151 Rennard SI, Locantore N, Delafont B, et al. Identification of five chronic obstructive pulmonary disease subgroups with different prognoses in the ECLIPSE cohort using cluster analysis. Ann Am Thorac Soc 2015; 12: 303-312.

152 Hastie AT, Martinez FJ, Curtis JL, et al. Association of sputum and blood eosinophil concentrations with clinical measures of COPD severity: an analysis of the SPIROMICS cohort. Lancet Respir Med 2017; 5: 956-967.

153 Lee JH, Cho MH, McDonald ML, et al. Phenotypic and genetic heterogeneity among subjects with mild airflow obstruction in COPDGene. Respir Med 2014; 108: 1469-1480.

154 Castaldi PJ, Dy J, Ross J, et al. Cluster analysis in the COPDGene study identifies subtypes of smokers with distinct patterns of airway disease and emphysema. Thorax 2014; 69: 415-422.

155 Reddel HK, Gerhardsson de Verdier M, Agustí A, et al. Prospective observational study in patients with obstructive lung disease: NOVELTY design. ERJ Open Res 2019; 5: 00036-02018. 